Canadian

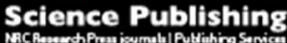

Biochemistry and Cell Biology

Biochimie et biologie cellulaire

\title{
Nucleolin and Nucleophosmin: nucleolar proteins with multiple functions in DNA repair
}

\begin{tabular}{|r|l|}
\hline Journal: & Biochemistry and Cell Biology \\
\hline Manuscript ID & bcb-2016-0068.R1 \\
\hline Manuscript Type: & Invited Review \\
\hline Date Submitted by the Author: & $15-$ May-2016 \\
\hline Complete List of Authors: & $\begin{array}{l}\text { Scott, Daniel; Institut de recherches cliniques de Montréal (IRCM), } \\
\text { Systems Biology; McGill University, Experimental Medicine } \\
\text { Oeffinger, Marlene; Institut de recherches cliniques de Montréal (IRCM), } \\
\text { Systems Biology; Universite de Montreal, biochimie et médecine } \\
\text { moléculaire; McGill University, Experimental Medicine }\end{array}$ \\
\hline Keyword: & Nucleolus, DNA repair, DNA damage, Nucleolin, Nucleophosmin \\
\hline \multicolumn{2}{|c}{} \\
\hline
\end{tabular}


Nucleolin and Nucleophosmin: nucleolar proteins with multiple functions in DNA repair

Daniel D. Scott ${ }^{1,2}$ and Marlene Oeffinger ${ }^{1,2,3, *}$

${ }^{1}$ Laboratory of RNP Biochemistry, Institut de recherches cliniques de Montréal (IRCM), Montréal,

Québec, H2W 1R7, Canada

${ }^{2}$ Division of Experimental Medicine, Faculty of Medicine, McGill University, Montréal, Québec, H3A 2A3, Canada

${ }^{3}$ Département de biochimie et médecine moléculaire, Faculté de Médecine, Université de Montréal, Québec, H3T 1J4, Canada

* To whom correspondence should be addressed: Tel (+1) 514987 5668; marlene.oeffinger@ircm.qc.ca 


\section{Abstract}

The nucleolus represents a highly multifunctional intranuclear organelle in which, in addition to the canonical ribosome assembly, numerous processes such as transcription, DNA repair and replication, the cell cycle and apoptosis are coordinated. The nucleolus is further a key hub in the sensing of cellular stress and undergoes major structural and compositional changes in response to cellular perturbations. Numerous nucleolar proteins have been identified that, upon nucleolar stress sensing, deploy additional, non-ribosomal roles in the regulation of varied cell processes including cell cycle arrest, arrest of DNA replication, induction of DNA repair, and apoptosis, among others. The highly abundant proteins nucleophosmin (NPM1) and nucleolin (NCL) are two such factors that transit to the nucleoplasm in response to stress, and participate directly in the repair of numerous different DNA damages. This review discusses the contributions made by NCL and/or NPM1 to the different DNA repair pathways employed by mammalian cells to repair DNA insults, and examines the implications of such activities for the regulation, pathogenesis and therapeutic targeting of NPM1 and NCL.

\section{Keywords}

Nucleolin; Nucleophosmin; Nucleolus; DNA repair; DNA damage 


\section{Introduction}

The nucleolus is a large, membraneless subnuclear organelle present in interphase cells throughout eukaryotes. It is a highly dynamic structure, undergoing disassembly and reassembly at each mitotic division and exhibiting major structural and compositional changes in response to growth signals, cellular status and stresses (Boulon et al. 2010). Electromicrographic studies have shown that nucleoli contain several distinct substructures, with the number and nature of these structures differing between species; anamniotic eukaryotes and the evolutionarily ancient turtles show a bipartite nucleolus, containing Fibrillar (F) and Granular (G) Zones, while the remaining amniotes exhibit a tripartite nucleolus containing a distinct Fibrillar Centre (FC), Dense Fibrillar Component (DFC) and Granular Component (GC; Lamaye et al. 2011; Thiry and Lafontaine 2005). Since the mid-1960s the nucleolus has been known as the site of ribosomal RNA (rRNA) transcription, processing and ribosomal assembly, and the different substructures within the nucleolus likely mediate distinct stages of the ribosome assembly process (Pederson 2011; Hernandez-Verdun et al. 2010). Several decades of research by many groups have made great strides towards elucidating the complicated succession of processing steps required to assemble nascent rRNA into functional ribosomes, as well as the identifying the $\sim 80$ ribosomal proteins (RPs) and >300 ribosomal maturation factors that are required to accomplish them (Henras et al. 2014; Venema and Tollervey, 1999; Woolford and Baserga 2013).

The evolution of increased structural complexity within the nucleolus in higher eukaryotes has coincided with a remarkable expansion of the size and complexity of the nucleolar proteome. In addition to the range of ribosome biogenesis factors above, the mammalian nucleolus contains a vast array of proteins with no known ribosomal function, many of which play important roles in the regulation of other cellular processes related to cell growth, proliferation and death (Andersen et al. 2002; Andersen et al. 2005; Leung et al. 2006). Indeed, more than 4,500 proteins have now been identified proteomically as exhibiting some degree of nucleolar localization, and many of these exhibit marked changes in their 
localization in response to cellular stresses such as DNA damage (Ahmad et al. 2009; Boisvert et al. 2010; Moore et al. 2011). Since these discoveries, our understanding of nucleolar functions has been revolutionized, and the nucleolus is now known to be a central hub for the coordination of numerous cellular processes including cell cycle control, apoptosis, mRNA maturation and regulation, transcription, DNA replication, and DNA repair (Boisvert et al. 2007). The nucleolus is particularly important for coordinating these varied pathways in response to a range of cellular stresses, and is now known to be a central hub for the sensing of stress and implementation of cellular responses (Boulon et al. 2010).

One particularly important role for the nucleolus is the coordination of ribosomal RNA transcription and ribosomal maturation with cellular growth and DNA replication/repair, and vice versa. The production of ribosomal RNA occupies $>50 \%$ of all eukaryotic transcription and represents the major source of cellular energy consumption, as well as being tightly coordinated with cell growth and division (Henras et al. 2014). As a result, any defect in ribosome biogenesis must be rapidly communicated to the cell's proliferative machinery to prevent division of cells with insufficient translational capacity; similarly, inhibition of ribosome synthesis in response to DNA damage or other stresses is essential to prevent cellular energy depletion and/or production of aberrant ribosomes from damaged rDNA (James et al. 2014; Tsai and Pederson 2014). Indeed, eukaryotic cells have developed an array of regulatory checks and balances to arrest rRNA transcription and processing under stress conditions (Boulon et al. 2010) and, conversely, to induce cell cycle arrest and/or apoptosis in response to perturbation of ribosome production (termed the 'nucleolar stress response'; Suzuki et al. 2012).

Interestingly, a burgeoning number of ribosomal and ribosome biogenesis proteins are being identified that go beyond this regulatory paradigm and participate directly in the repair of damaged DNA in vivo. RPS3 (a.k.a. uS3) has been reported to act as an apurinic/apyrimidinic endonuclease in both nuclear and mitochondrial base excision repair (Kim et al. 2013), while nucleostemin/GNL3 has been implicated in the recruitment of RAD51 to stalled replication forks (Lin et al. 2013; Meng et al. 2013), and Nol12 has 
been identified as an important protective factor against oxidative DNA damage (Scott et al. 2016). Most strikingly, two proteins - nucleophosmin (NPM1) and nucleolin (NCL) - are required for optimal activity of multiple independent DNA repair pathways, and their dysregulation has been reported in numerous cancers, in particular leukemias and other myeloproliferative disorders (Berger et al. 2015; Colombo et al. 2011; Federici et al. 2013).

In this review, we will discuss in detail the multifaceted functions of NPM1 and NCL in the resolution of DNA damage; in particular, how NPM1 and NCL are able to interact with and directly modulate the different DNA repair systems of eukaryotic cells including double-strand break repair by homologous recombination (HR) or non-homologous end joining (NHEJ), base excision repair (BER), nucleotide excision repair (NER) and translesion synthesis (TLS). We will also consider how NPM1 and NCL are able to coordinate the activities of key DNA repair factors such as APE1, RPA and p53, and how these modulations may contribute to cellular DNA repair activities. Finally, we will discuss some of the key concepts relating to the DDR functions of NPM1 and NCL, and how these two proteins may contribute to the complicated and inter-related pathways controlling cellular proliferation and recovery in response to stress stimuli.

\section{Nucleophosmin and Nucleolin: two multifunctional nucleolar proteins}

Nucleophosmin (NPM1, also known as numatrin, NO38 or NPM) and nucleolin (NCL) were first identified by Busch and colleagues in 1973, where they were determined as spots B23 and C23 respectively in 2D gel electrophoreses of purified nucleoli (Orrick et al. 1973). The two proteins represent major constituents of mammalian nucleoli, with NCL in particular comprising up to $10 \%$ of all protein content within the nucleolus (Durut and Saez-Vasquez 2015). Subsequent work has shown that these two proteins share numerous physiological features including their ability to undergo nucleocytoplasmic shuttling (Borer et al. 1989) and to act as RNA and protein chaperones (Angelov et al. 2006; Borggrefe et 
al. 1998; Hanakahi et al. 2000; Szebeni and Olson 1999; Thyagarajan et al. 1998), their active relocalization from the nucleolus to the nucleoplasm in response cellular stresses (Colombo et al. 2011; Tajrishi et al. 2011), and their ability to interact with each other, among others (Li et al. 1996). Several decades of detailed functional study have revealed that both proteins are remarkably multifunctional, playing overlapping but non-identical roles in a range of cellular survival and proliferation pathways including ribosome biogenesis, cell cycle and apoptosis, transcriptional regulation, and DNA replication and repair (Colombo et al. 2011; Tajrishi et al. 2011). In this section, we will discuss the structure and regulation of NPM1 and NCL, and will briefly introduce their functions outside of the DDR machineries.

The multifaceted roles of NPM1 outside of DNA damage response pathways

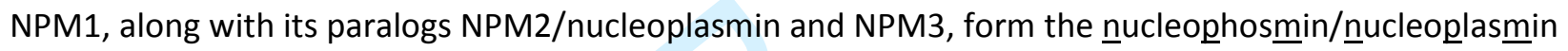
(NPM) family of proteins. Each of these three proteins is conserved throughout vertebrates, while more evolutionarily distant members of the family may be found in invertebrates and in lower eukaryotes (Edlich-Muth et al. 2015; Frehlick et al. 2007; Gudavicius et al. 2014), and all three possess the ability to bind histones, regulate their assembly into nucleosomes and promote their loading onto and/or eviction from DNA, identifying them as true histone chaperones (Frehlick et al. 2007; Finn et al. 2012). While NPM2 and NPM3 appear in vivo to function exclusively as histone chaperones during both somatic cell growth and, in particular, gametogenesis and fertilization (Finn et al. 2012; Okuwaki et al. 2012), NPM1 has gained numerous additional functions during evolution and acts in numerous independent cellular pathways. The NPM1 gene encodes two protein isoforms, termed B23.1 (294 amino acids) and B23.2 (259 amino acids), which differ by splicing at their C-termini (Colombo et al. 2011). The B23.2 isoform is poorly expressed in mammalian cells and has not been extensively investigated; work discussed herein will focus on the predominant B23.1 isoform. 
Mammalian NPM family members share a common structural arrangement, typically consisting of an oligomerization domain at the $\mathrm{N}$-terminal end of the protein, a central/C-terminal nuclear localization signal, and several central acidic tracts containing predominantly or exclusively aspartate and/or glutamate residues (Colombo et al. 2011; Hingorani et al. 2000). NPM1 additionally contains nuclear export signals (NESs), supporting its reported nucleocytoplasmic shuttling activity, and an extended Cterminal tail responsible for many of its chaperone-independent functions (see Fig. 1; Hingorani et al. 2000; Wang et al. 2005; Yu et al. 2006; Borer et al. 1989; Colombo et al. 2011). In some family members from lower eukaryotes, the canonical NPM-family oligomerization domain is fused with a peptidyl-prolyl isomerase domain orthologous to that of mammalian FKBP25, a known NPM1/NCL interactor (EdlichMuth et al. 2015; Gudavicius et al. 2014). Structural and biochemical investigations have shown that both NPM1 and NPM2 can assemble into a large, homopentameric ring structure through its oligomerization domain (Dutta et al. 2001; Lee et al. 2007; Mitrea et al. 2014; Namboodiri et al. 2004, 2003; Platonova et al. 2011). While several crystallographic studies have described decameric (i.e. headto-head double-pentameric) structures for NPM1 family members, these decamers are highly structurally divergent from one another (Dutta et al. 2001; Lee et al. 2007; Namboodiri et al. 2003; Namboodiri et al. 2004) and are not observed in biophysical studies of soluble protein (Franco et al. 2008; Mitrea et al. 2014; Taneva et al. 2009). The oligomerization of NPM1 is essential for at least some of its functions in vivo, likely through allowing simultaneous and closely juxtaposed binding of multiple targets or of multi-subunit complexes (Thyagarajan et al. 1998; Xia et al. 2013). Interestingly, NPM3 has not been reported to form homopentameric rings and is unable to act as a histone chaperone in its own right; instead, it assembles into hetero-oligomers with NPM1, in doing so promoting the histone chaperone activity of NPM1 while repressing its RNA-binding and ribosome biogenesis activities (Finn et al. 2012; Huang et al. 2005; Okuwaki et al. 2012). 
Consistent with its identification as a nucleolar phosphoprotein, NPM1 undergoes extensive posttranslational modifications including phosphorylation, ubiquitination and SUMOylation, arginine methylation, lysine acetylation and others (Colombo et al. 2011), demonstrating it to be a highly regulated protein in vivo. Immunofluorescence studies have confirmed early observations that NPM1 is a predominantly nucleolar protein in unstressed cells; however, this localization is highly dynamic in response to cellular stresses. NPM1 undergoes rapid and reversible translocation from the nucleolus to the nucleoplasm (nucleoplasmic translocation) upon exposure to UV irradiation, oxidative stress, heat shock or numerous DNA-damaging agents including mitomycin C, methyl methanesulfonate (MMS) and cisplatin; however, NPM1 does not alter its localization in response to ionizing radiation (IR) or exposure to double strand break (DSB)-inducing topoisomerase inhibitors such as etoposide or camptothecin (Kurki et al. 2004; Vanderwaal et al. 2009; Yogev et al. 2008). Importantly, these localization changes do not coincide with relocalization of $\mathrm{NCL}$ or a generalized dissociation of the nucleolus as seen in response to RNA Pol I inhibition, and evidence is emerging for their control by particular, DNA-damage-responsive signaling pathways and/or post-translational modifications (Kurki et al. 2004; Liu et al. 2007; Poletto et al. 2014; Sato et al. 2004; Vanderwaal et al. 2009; Yogev et al. 2008). Given such evidence, it is likely that the nucleoplasmic translocation of NPM1 is an active and controlled response to particular genotoxic stress conditions and not the result of generalized nucleolar disruption.

NPM1 possesses a wide range of biochemical activities. It was initially described as a molecular chaperone, which binds histones and other small, positively charged proteins such as ribosomal proteins (RPs) via its central acidic tracts (Okuwaki et al. 2001; Szebeni and Olson 1999; Yu et al. 2006). As a histone chaperone, NPM1 binds most efficiently to acetylated $\mathrm{H} 3 / \mathrm{H} 4$ heterodimers and, as mentioned above, is able to promote the activities of nucleosome remodeling complexes and to support transcription and chromatin packaging activities (Okuwaki et al. 2001; Swaminathan et al. 2005). The ability of NPM1 to interact with highly positive sequences, along with its oligomerization activity have 
led several groups to propose that NPM1 may be the scaffolding protein responsible for retention of proteins containing arginine/lysine-rich nucleolar localization signals (NoLSs) within the nucleolus (Emmott and Hiscox 2009; Li et al. 1996; Mitrea et al. 2016). Through its C-terminal regions, NPM1 is able to bind short ssDNA sequences and promote dsDNA strand annealing (Borggrefe et al. 1998) and, independently of those functions, to also interact with structured RNAs in response to genotoxic stress (Yang et al. 2002). Finally, NPM1 possesses RNA-specific endonuclease activity mediated by regions within its C-terminus (Herrera et al. 1995; Savkur and Olson 1998).

As expected for a predominantly nucleolar protein, NPM1 has long been considered a key ribosome biogenesis factor, and indeed NPM1 regulates the synthesis and maturation of ribosomes at several levels. Firstly, NPM1 is able to directly associate with rDNA repeats within the nucleolus and promote their transcription, potentially through its histone chaperone activities (Murano et al. 2008). Secondly, NPM1 was found to associate with pre-rRNA within the nucleolus and mediate endonucleolytic cleavage within the Internal Transcribed Spacer 2 (ITS2), thereby separating the $28 \mathrm{~S}$ and 5.8S rRNAs (Savkur and Olson 1998). Thirdly, NPM1 is able to bind to assembled 40S and 60S ribosomal subunits and promote their nuclear export via the CRM1 pathway, acting as a nucleocytoplasmic chaperone (Maggi et al. 2008; Yu et al. 2006). In addition to these roles, NPM1 possesses numerous nonribosomal functions. NPM1 binds to and regulates the activity of a range of numerous transcription factors including p53, c-Myc, androgen receptor, Miz1, NFKB, AP2 $\alpha$ and CRCF, as well as regulating global RNA Pol II transcription through an interaction with HEXIM1 (Colombo et al. 2011; Gurumurthy et al. 2008). In conjunction with this transcriptional regulation of expression, NPM1 may act post-transcriptionally through loading onto mRNAs during polyadenylation and, possibly, promotion of their processing within nuclear speckles (Palaniswamy et al. 2006; Tarapore et al. 2006). In B cell lineages, NPM1 has been found in a complex with NCL, PARP1 and SWAP70 (termed the SWAP complex), which promotes the recombination of $I g H$ gene switch regions (Borggrefe et al. 1998). Finally, NPM1 was found to be recruited to centrosomes in a 
manner dependent on its oligomerization and interaction with BRCA2 and ROCK2 (Wang et al. 2011; Xia et al. 2013). This recruitment stabilizes centrosomes and inhibits their duplication; release of NPM1 from centrosomes due to T199 phosphorylation by CDK2/cyclin E at the G1/S boundary licenses centrosomal replication during S-phase (Okuda et al. 2000; Tarapore et al. 2002; Tokuyama et al. 2001). Constitutive dissociation of NPM1 from centrosomes, due to overexpression of free binding partners or inhibition of oligomerization, results in aberrant centrosomal duplication, chromosomal instability and the formation of multinucleate cells (Wang et al. 2011; Xia et al. 2013), suggesting that the control of centrosomal duplication by NPM1 may be an important mechanism for ensuring genomic stability during mitosis. Interestingly, the observation that NPM1 is found in a complex with CENP-A, a protein that is substituted for histone $\mathrm{H} 3$ in centrosomal nucleosomes, suggests that the histone chaperone activity of NPM1 may be important for its regulation of centrosomal replication (Foltz et al. 2009). The collective significance of these numerous functions of NPM1 are underlined by the observation that mouse models of both NPM1 haploinsufficiency $\left(N P M 1^{+-}\right)$and hypomorphism $\left(N P M 1^{h m / h m}\right)$ are embryonically lethal and exhibit numerous severe neurological and hematological developmental failures prior to termination (Grisendi et al. 2005).

NCL throughout the cell - nucleolus, nucleoplasm, cytoplasm and cell surface

$\mathrm{NCL}$ is a highly abundant $77 \mathrm{kDa}$ phosphoprotein, the most abundant nucleolar protein in mammals and the major silver-binding component of argyrophilic nucleolar organizing regions (AgNORs; Tajrishi et al. 2011). Unlike NPM1, NCL is conserved throughout eukaryotes, with homologs observed in plants, animals and yeast (S. cerevisiae Nsr1p). NCL shares certain structural features with NPM1, including three central acidic aspartate/glutamate-rich stretches and a bipartite NLS (Schmidt-Zachmann and Nigg 1993), but unlike NPM1, it possesses a highly ordered, modular domain arrangement with an N-terminal 
$\left(\mathrm{X}-\mathrm{T}-\mathrm{P}-\mathrm{X}-\mathrm{K}_{2}-\mathrm{X}_{2}\right)_{8}$ phosphorylated repeat region, four successive RNA binding domains (RBDs) and an extreme C-terminal arginine/glycine rich region (RGG/GAR domain, see Fig. 1; Ginisty et al. 1999; Tajrishi et al. 2011). While the structures of individual NCL domains have been determined, the full protein has thus far eluded structural mapping. NCL undergoes extensive post-translational modification by phosphorylation, acetylation, methylation and ubiquitination, as well as being proteolytically cleaved into independently active sub-forms under certain conditions, which were shown to fulfill different cellular functions (Chen et al. 1991; Tajrishi et al. 2011; Warrener and Petryshyn 1991). Like NPM1, NCL is a predominantly nucleolar protein under non-stress conditions, though minor but biologically distinct populations have been observed in the nucleoplasm, cytoplasm and at the cell surface in certain cell types/tissues. Also like NPM1, NCL exhibits rapid, reversible and post-translationally-regulated nucleoplasmic translocation in response to exposure to genotoxic stress; however, NCL responds to a very different suite of genotoxic conditions than NPM1, exhibiting robust translocation upon exposure to IR, DSB-inducing agents such as camptothecin and etoposide, and heat shock. In contrast, NCL does not alter its localization upon exposure to UV irradiation, oxidative stress or nucleotide/base-altering agents such as MMS and bleomycin; controversy exists regarding NCL's relocalization in response to replication stress (Daniely and Borowiec 2000; Daniely et al. 2002; Kim et al. 2005; Indig et al. 2012; Kobayashi et al. 2012). Importantly, these observations demonstrate that, while NPM1 and NCL may share a physiological response to stress conditions, their individual activating stressors are very different, providing cells with overlapping and complementary stress sensor systems.

Biochemically, NCL is able to act as a histone chaperone through its central acidic tracts, thereby supporting chromatin remodeling by the SWI/SNF and ACF complexes in a manner similar to that of NPM1. Unlike NPM1, however, NCL interacts specifically with the H2A-H2B histone dimer (Angelov et al. 2006). NCL has been reported to support the eviction of $\mathrm{H} 2 \mathrm{~A}-\mathrm{H} 2 \mathrm{~B}$ dimers from histone octamers, an important process for DNA transcription, replication and repair (Angelov et al. 2006; Kobayashi et al. 
2012). NCL can also act on the variant histone macro-H2A1, which is loaded onto transcriptionally silent rDNA repeats (Angelov et al. 2006; Cong et al. 2012). NCL has also been reported to possess protease activity responsible for phosphorylation-sensitive autoproteolysis under certain conditions (Chen et al. 1991; Fang and Yeh 1993; Warrener and Petryshyn 1991), though others have attributed this proteolysis to other cellular proteases (Hsu et al. 2015; Pasternack et al. 1991). NCL possesses both strandannealing activity for complementary and partially complementary ssDNA sequences (Hanakahi et al. 2000; Thyagarajan et al. 1998) and, according to some reports, DNA/RNA helicase activity (Hanakahi et al. 2000; Seinsoth et al. 2003; Tuteja et al. 1995). Finally, NCL possesses stress-responsive RNA-binding activity, mediating interaction of NCL with a number of mRNAs in vivo (Yang et al. 2002).

NCL's predominantly nucleolar localization directed early functional studies towards the regulation of ribosome biogenesis. NCL binds directly to rDNA in vivo and is required for the loading of general transcription factors (TFs) and, ultimately, progression of RNA Pol I along the gene (Cong et al. 2012; Rickards et al. 2007). In this context, NCL contributes extensively to the remodeling of rDNA chromatin in vivo, likely through its roles as a $\mathrm{H} 2 \mathrm{~A}-\mathrm{H} 2 \mathrm{~B}$ and/or macro-H2A1 histone chaperone (Durut and SaezVasquez 2015; Cong et al. 2012). Independently, NCL binds directly to nascent 475 pre-rRNA transcripts

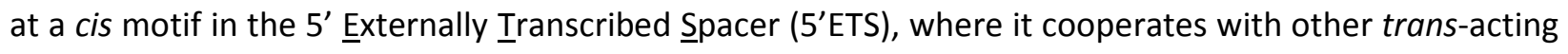
factors, in particular the U3 snoRNP, to promote the first endonucleolytic cleavage of the 47S pre-rRNA (Ginisty et al. 2000; Turner et al. 2009). Outside of the nucleolus, NCL is able to bind and modulate the activity of several TFs in response to cellular stresses (Tajrishi et al. 2011). NCL can also interact directly with DNA at G-quadruplex structures through its terminal two RBDs and its RGG domain, stabilizing these structures in vitro and in vivo (González and Hurley 2010; Hanakahi et al. 1999; Indig et al. 2012). This activity mediates the binding of NCL in vivo to numerous G-rich DNA sequences within ribosomal DNA (rDNA) genes, IgH coding regions and telomeric repeats (González and Hurley 2010; Indig et al. 2012; Ishikawa et al. 1993) and enables it to act directly as a transcriptional regulator at these sites. For 
example, NCL has been reported to cooperate with hnRNP D to form the heterodimeric LR1 Gquadruplex-binding complex that transcriptionally activates IgH sequences in B-cells (Dempsey et al. 1998; Hanakahi et al. 1997). In contrast, NCL binding to and stabilization of a defined G-quadruplex structure within the $c-M Y C$ gene promoter contributes to transcriptional repression of this locus, possibly by abolishing binding sites for activating TFs or altering the local chromatin (González et al. 2009; González and Hurley 2010). Post-transcriptionally, NCL binding to mRNAs may effect expression of particular proteins, with NCL binding to the $p 53$ mRNA 5'UTR resulting in translational activation, while its binding to gadd45 $\alpha$ mRNA in response to arsenite treatment and K294-SUMOylation blocks the degradation of this transcript (Takagi et al. 2005; Zhang et al. 2015). Remarkably, NCL has even been reported to be constitutively displayed on the cell surface, where it may act as a ligand for particular growth-regulatory ligands and viruses (Fujiki et al. 2014; Hovanessian et al. 2010; Nisole et al. 2002).

\section{Regulating the regulator: NCL and NPM1 promote p53 stress-dependent stabilization}

p53 is a master regulator of cell proliferation, growth and death, acting as a key hub in the communication of cellular stresses to downstream targets through transcriptional, post-transcriptional and post-translational means (Bieging et al. 2014). p53 is consequently a key tumor suppressor in humans, and more than $50 \%$ of all human tumors carry mutations in the TP53 gene. A key regulator of p53 itself is HDM2 (MDM2 in mice), an E3 ubiquitin ligase that interacts with p53 in unstressed cells and promotes p53 ubiquitination and proteasomal turnover. Disruption of this interaction, and consequent stabilization of p53 protein, is a major mechanism by which stress signals communicate via p53 (Wade et al. 2013).

In response to activating cellular stresses, both NCL and NPM1 are able to modulate the p53-HDM2 axis to induce p53 stabilization and promote its tumor-suppressive functions. NPM1 interacts directly with 
$\mathrm{p} 19^{\mathrm{ARF}}$ ( $\mathrm{p} 14^{\mathrm{ARF}}$ in mice), an important tumor suppressor transcribed from the same gene as the p16 ${ }^{\text {INK4a }}$ cyclin-dependent kinase inhibitor but translated from an alternative reading frame (ARF). In unstressed cells, the NPM1-p19ARF interaction is constitutive, causing sequestration of $\mathrm{p} 19^{\text {ARF }}$ into the nucleolus where it is unable to act on p53-HDM2. Upon exposure to an NPM1-activating cellular stress, the interaction between NPM1 and $\mathrm{p} 19^{\mathrm{ARF}}$ is disrupted, resulting in mobilization of $\mathrm{p} 19^{\mathrm{ARF}}$ to the nucleoplasm, where it interacts with HDM2 and interferes with p53-HDM2 complex formation, as a result stabilizing p53 (Gjerset 2006). Interestingly, it has been reported that, in addition to this pathway, NPM1 is able to interact with HDM2 directly in a fashion that competes out p53-HDM2 interactions, stabilizing p53 (Kurki et al. 2004). Unlike the canonical nucleolar stress pathway in which GLTSCR2/PICT1, RPL11/RPL5 (a.k.a. uL5/uL18, respectively) and 5S ribosomal RNA cooperate to sequester HDM2 in the nucleolus following inhibition of ribosome biogenesis, the NPM1-HDM2 interaction does not induce HDM2 relocalization. Instead, NPM1 that has exited the nucleolus appears to directly interrupt HDM2 binding to p53, preventing ubiquitination and consequent degradation of p53. (Kurki et al. 2004; Suzuki et al. 2012). NPM1 has also been reported to interact directly with p53 itself in order to drive p53 stabilization (Colombo et al. 2002), though this finding has been placed into question by a report showing an association of NPM1 only with a poly-SUMOylated form of p53 (Kurki et al. 2004). These different mechanisms likely represent ways by which NPM1 can reinforce the $\mathrm{p} 19^{\mathrm{ARF}} / \mathrm{HDM} 2 / \mathrm{p} 53$ pathway and thus protect against mutagenic events.

NCL has been reported to interact with both HDM2 and p53 (Daniely et al. 2002; Saxena et al. 2006). However, domain-mapping experiments suggest that NCL binds $\mathrm{p} 53$ and HDM2 via different regions the C-terminal RGG domain and N-terminal RBD domains, respectively - and may form a ternary complex with both proteins simultaneously (Bhatt et al. 2012). While NCL's interaction with HDM2 has been shown to inhibit HDM2-mediated p53 destabilization, both through driving p53-HDM2 dissociation and by promoting HDM2's own ubiquitination and degradation (Bhatt et al. 2012; Saxena et al. 2006), 
the formation of an NCL-p53-HDM2 ternary complex was also shown to result in recruitment of a ubiquitin protease, HAUSP, which supports deubiquitination and stabilization of p53, HDM2 and NCL (Lim et al. 2015). How these apparently contradictory pathways may be coordinated and/or interact to mediate NCL-dependent p53 stabilization remains to be fully dissected. As well as modulating p53 stabilization, the p53-NCL interaction was also shown to be required for nucleoplasmic translocation of $\mathrm{NCL}$ in response to cellular stresses such as heat shock, IR and camptothecin treatments, although other signals are likely required to facilitate NCL-p53 interactions under these conditions (Daniely et al. 2002; Kim et al. 2005).

\section{NPM1 and NCL in histone remodeling and double strand break repair}

Among DNA damages, double-strand breaks (DSBs) are the most deleterious due to their ability to cause major rearrangements of chromosomes and the difficulty of repairing two free, non-juxtaposed DNA ends. DSBs can arise from numerous sources in vivo, including directly from chemical or irradiative insults on DNA, or from fragmentation of fragile DNA structures such as stalled replication forks, base/nucleotide damages, or nucleosome-free regions (Kowalczykowski 2015). Consequently, cells have developed two major, overlapping repair systems for the detection, management and repair of DSBs at different stages of the cell cycle. The first is homologous recombination (HR), in which free DNA ends are exonucleolytically resected and a complementary sister chromatid is used as a template for synthesis across the break point prior to end rejoining (Kowalczykowski 2015). The dependence on a sister chromatid as a template means that HR is accomplished exclusively in late-S and G2/M phase but is highly accurate, rarely resulting in mutations (Kakarougkas and Jeggo 2014). The second method, nonhomologous end joining (NHEJ), involves direct juxtaposition and relegation of free DNA ends, providing a more efficient, but more error-prone, process (Ochi et al. 2014). Unlike HR, NHEJ occurs throughout the cell cycle and is responsible for the majority of DSB repair events occurring in vivo (Kakarougkas and 
Jeggo 2014). While the machineries that accomplish HR and NHEJ are different and highly complex, both require initial recognition of the DSB and propagation a pro-repair signal across up to several megabases $(\mathrm{Mb})$ of DNA on either side of the break, allowing large-scale recruitment of downstream regulatory and repair factors. The size of these labeled and regulated DNA regions allows the visualization of individual DSB repair foci by microscopy via immunofluorescent detection of DSB-associated factors or posttranslational modifications.

The observation that $\mathrm{NCL}$ undergoes robust yet reversible nucleoplasmic translocation in response to stress stimuli such as IR, camptothecin and etoposide treatment - each of which induces DSB formation - suggests that NCL may participate in the cellular response to DSB damage (Daniely et al. 2002; Indig et al. 2012; Kobayashi et al. 2012). Indeed, several groups have observed that in IR- or camptothecintreated cells NCL forms nucleoplasmic foci that colocalize with sites of DSB repair, coinciding temporally with the formation of DSBs (Indig et al. 2012; Kobayashi et al. 2012), and returns to the nucleolus upon their clearance (Goldstein et al. 2013; Kobayashi et al. 2012), supporting the conclusion that NCL plays a direct role in the repair and clearance of DSBs. Recent studies have begun to provide detailed insights into the potential role of $\mathrm{NCL}$ at DSBs in vivo. Two groups in particular undertook detailed functional characterization of DSB repair in the absence of $\mathrm{NCL}$ and found that, while $\mathrm{NCL}$ depletion does not compromise the formation of repair foci upon genotoxic stress, it does compromise their clearance after the stress is removed. Such a phenotype indicates the occurrence of specific defect(s) in later stage(s) of the DSB repair process that prevent completion of DSB repair and, consequently, persistence of 'stalled' repair foci (Goldstein et al. 2013; Kobayashi et al. 2012). Further assays confirmed that depletion of NCL, either by antibody microinjection or siRNA knockdown $(\mathrm{kd})$, did not inhibit recruitment of early DSB repair factors such as the Mre11-Rad50-Nbs1 (MRN) complex or $\mathrm{yH} 2 \mathrm{AX}$, but did cause a failure to repair DSBs by both HR and NHEJ (De et al. 2006; Goldstein et al. 2013; Kobayashi et al. 2012). Using a ChIPbased assay on a site-specific, NHEJ-targeted DSB, Goldstein et al. (2013) demonstrated that NCL is 
recruited to sites of DSB repair through an interaction with the Rad50 subunit of the MRN complex, and that while this recruitment has no effect on the loading of DSB repair factors such as $\gamma H 2 A X, A T M$, MDC1, RNF8 and RNF168, it did coincide with the rapid depletion of $\mathrm{H} 2 \mathrm{~A}-\mathrm{H} 2 \mathrm{~B}$, but not $\mathrm{H} 3-\mathrm{H} 4$, dimers within a few kilobases of the DSB site. Kobayashi and colleagues similarly observed reduced $\mathrm{H} 2 \mathrm{~A}-\mathrm{H} 2 \mathrm{~B}$ mobilization from chromatin to the nucleoplasm upon depletion of NCL (Kobayashi et al. 2012). These observations echo findings that NCL facilitates $\mathrm{H} 2 \mathrm{~A}-\mathrm{H} 2 \mathrm{~B}$ eviction at transcriptional loci by the chromatin remodeling complexes SWI/SNF and ACF, both of which have also been found to contribute to DSB repair (Angelov et al. 2006; Bao 2011; Lan et al. 2010). Interestingly, Kobayashi et al. additionally observed that, i) NCL was able to interact directly with the DSB repair factor MDC1; ii) NCL depletion prior to IR exposure reduced the partitioning of MDC1 into a chromatin-containing subcellular fraction; and iii) NCL depletion resulted in reduced formation of DSB repair foci containing RNF168, an H2A/H2AX-ubiquitin E3 ligase recruited by MDC1 (Kobayashi et al. 2012). This finding remains contentious - they themselves found that, despite the reduced chromatin partitioning of MDC1 upon NCL depletion, immunofluorescent localization of MDC1 to DSB foci was unaffected, while Goldstein and colleagues' ChIP-based assay found no reduction in the recruitment of MDC1 to a defined DSB site in the absence of NCL (Goldstein et al. 2013). It is known, however, that RNF8/RNF168-dependent H2A/H2B ubiquitination is an important activating step in histone eviction both following DNA damage and during spermiogenesis, in which histone octamers are globally evicted from DNA and replaced by protamines to facilitate greater DNA compaction; such observations suggest that regulation of MDC1 and its partners by NCL could conceivably play a role in NCL's histone-chaperone activity at DSBs (Ikura et al. 2007, Lu et al. 2010, Rathke et al. 2014). Collectively, these data argue for a model in which NCL recruitment to DSBs via the MRN complex facilitates the proximal nucleosome destabilization by chromatin remodeling complexes, possibly through the actions of MDC1 and its targets RNF8/RNF168, and ultimately facilitates NHEJ- and HR-mediated DSB resolution (Goldstein et al. 2013). 
While these two works present the most complete model of the role of NCL at DSBs, several other groups have described alternative, not mutually exclusive models that may also contribute to $\mathrm{NCL}$ activity at DSB sites. NCL has been reported to interact directly with WRN, a dsDNA helicase and exonuclease responsible for resolving paired DNA structures that may form at sites of DNA repair or replication, and was shown to colocalize with WRN at a subset of IR-induced DSB foci. NCL binding to WRN was found to inhibit WRN's helicase but not exonuclease activity in vitro (Indig et al. 2012). NCL has also been found to coimmunoprecipitate (coIP) with RAD51, a key recombinase that is loaded onto ssDNA in place of RPA at sites of HR-mediated DSB repair, and to support HR only in the presence of RAD51 (De et al. 2006). While the contribution of this link to HR-mediated DSB repair in vivo has not been explored, it is important to note that RAD51 also interacts with and displaces RPA, another NCL interactor, on SSDNA, and that NCL has previously been found to support recombination as part of the B lymphocyte-specific SWAP complex (Borggrefe et al. 1998), suggesting that a possible role for the NCLRAD51 interaction in HR is not infeasible.

Although available data is less extensive than for $\mathrm{NCL}$, several lines of evidence point towards a role for NPM1 in the management of DSB repair. A combined subcellular fractionation-proteomics approach showed that NPM1 associated with chromatin in response to treatment of cells with IR or etoposide (Lee et al. 2005), despite the fact that nucleoplasmic translocation of this protein was not observed in response to these stimuli (Yogev et al. 2008). A subsequent study found that, while NPM1 could not be detected at DSB foci with an antibody against the whole protein, phosphorylation-specific antibodies revealed a minor, T199-phosphorylated population of NPM1 at these sites (see Fig. 1; Koike et al. 2010). This study demonstrated further that NPM1-pT199 is recruited to DSBs via interaction with nondegradative, K48-linked polyubiquitin chains generated by RNF8/RNF168 at DSB sites, and that this recruitment utilizes a novel ubiquitin-interacting motif-like (UIML) sequence within NPM1 which overlaps the third acidic tract and the T199 phosphosite (Koike et al. 2010). Expression of a T199A non- 
phosphorylatable mutant of NPM1 in vivo did not compromise DSB foci formation or recruitment of repair factors, but did result in a failure to religate DSBs and clear DSB foci at $6 \mathrm{~h}$ after irradiation, demonstrating that loss of NPM1 recruitment to DSBs is phenotypically similar to that of NCL (Goldstein et al. 2013; Kobayashi et al. 2012; Koike et al. 2010). The observation that NCL mediates DSB-proximal eviction of $\mathrm{H} 2 \mathrm{~A} / \mathrm{H} 2 \mathrm{AX}$, both polyubiquitination targets of RNF8/RNF168, suggests that NCL may exhibit a degree of epistasis over NPM1 in this system. While the biological function, if any exists, of NPM1 at DSBs has not yet been determined, it is interesting to note that ChIP-based analyses of DSB sites in cycling cells (in which both HR and NHEJ co-occur) allowed Goldstein et al. (2013) to demonstrate that $\mathrm{HR}$ requires the eviction not only of $\mathrm{H} 2 \mathrm{~A}-\mathrm{H} 2 \mathrm{~B}$ histone dimers by $\mathrm{NCL}$, but also the eviction of $\mathrm{H} 3-\mathrm{H} 4$ histones prior to end resection and RPA loading. Given that NPM1 has previously been suggested to possess H3-H4 chaperone activity (Okuwaki et al. 2001; Swaminathan et al. 2005), these observations raise the intriguing possibility that NPM1 recruitment to DSBs may be the mechanism by which this additional $\mathrm{H} 3-\mathrm{H} 4$ eviction is achieved, potentially identifying $\mathrm{NCL}$ and NPM1 as complementary histone chaperones at DSBs. The observation that T199 phosphorylation is performed by CDK2/cyclin E at the G1/S boundary (Okuda et al. 2000; Tokuyama et al. 2001; Tarapore et al. 2002) provides a possible explanation for the licensing of NPM1 activity at DSBs, which occurs in late S/G2-specific HR reactions (Goldstein et al. 2013). While this hypothesis is compelling, it should be noted that Goldstein et al. (2013) did find a partial abrogation of H3-H4 eviction in their assay following depletion of ASF1, a separate $\mathrm{H} 3-\mathrm{H} 4$ histone chaperone with roles in nucleosome reassembly after UV irradiation in humans (Battu et al. 2011) and DSBs in S. cerevisiae (Chen et al. 2008; Kim and Haber 2009), suggesting that if NPM1 does act at DSBs, it may share this activity with other histone chaperones. In addition, others have reported contrary activities for NPM1, identifying the protein as a "shielding" factor that blocks DSB repair in heat-shocked cells, possibly through tethering of DSB-containing DNA to the nuclear matrix (Vanderwaal et al. 2009; Vanderwaal and Roti Roti 2004). How these apparently contradictory 
observations can be resolved with the above model of NPM1 function within DSB repair remains to be seen.

\section{Holding out: NCL sequesters RPA away from the replication and/or repair machinery}

Replication Protein A (RPA) is an abundant, heterotrimeric complex consisting of three subunits - RPA1 (70 kDa), RPA2 (29 kDa) and RPA3 (14 kDa) - in humans. RPA is the predominant ssDNA-binding moiety in vertebrates, homologous to bacterial SSB protein (Prakash and Borgstahl 2012). In this role, RPA participates as a chaperone for and protector of ssDNA intermediates within a number of different DNAmetabolic processes including HR, nucleotide excision repair (NER), resolution of stalled replication forks (RFs), and lagging strand synthesis during DNA replication. As a consequence, RPA has a highly complex interactome, including proteins essential to all these pathways, and colocalizes with both DNA replication centers and DNA damage repair foci depending on the genotoxic load on the cell (Vassin et al. 2004).

$\mathrm{NCL}$ was initially identified as an RPA interactor through a biochemical screen for proteins retained on an ssDNA-RPA column (Daniely and Borowiec 2000), and was reported to interact with the RPA3 subunit in isolation; however, subsequent work reported that this interaction, mediated by the NCL RGG domain, may allow binding of NCL to the whole RPA complex (Kim et al. 2005; Wang et al. 2001). The interaction of $\mathrm{NCL}$ and RPA was found to be enhanced by exposure of cells to a range of stresses including heat shock, camptothecin, IR and $\mathrm{HU}$, and to predominantly occur in the nucleoplasm following translocation of NCL (Daniely and Borowiec 2000; Kim et al. 2005; Wang et al. 2001). The $\mathrm{NCL} / \mathrm{RPA}$ interaction - and nucleoplasmic translocation of $\mathrm{NCL}$ - could be rendered constitutive through phosphorylation-blocking mutagenesis of three proposed casein kinase II (CKII) phosphorylation sites

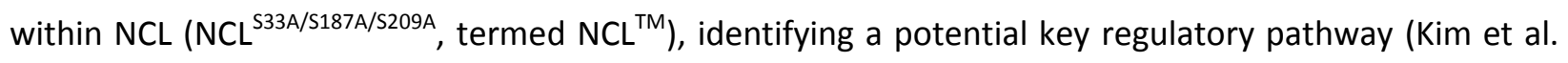


2005). Interestingly, however, the interaction of NCL/RPA and their immunofluorescent colocalization were not the result of NCL recruitment to sites of RPA activity. Instead, NCL-RPA complex formation coincided with a migration of RPA away from foci of DNA replication and into a cellular fraction containing aggregated and/or poorly-soluble proteins, suggestive of a sequestration-based, inhibitory role for NCL on RPA (Daniely and Borowiec 2000; Wang et al. 2001). Consistent with this, functional assays found that NCL was able to inhibit RPA-dependent SV40 origin unwinding by LTAg in vitro and that, in vivo, NCL-RPA interaction induced DNA replication arrest following heat shock or camptothecin treatment (Daniely and Borowiec 2000; Kim et al. 2005; Wang et al. 2001). Importantly, expression of the constitutive RPA-binding $\mathrm{NCL}^{\mathrm{TM}}$ mutant was able to induce replication arrest in the absence of genotoxic stress; this arrest could be rescued by simultaneous overexpression of RPA, confirming that this arrest is a direct result of NCL's sequestration of RPA rather than an indirect effect of other NCL functions (Kim et al. 2005).

One interesting question remains whether this sequestration of RPA by NCL is able to compromise DNA damage repair pathways that require RPA, such as HR and NER, as well as DNA replication. While the sequestration of RPA from replication centers is likely to reduce DNA replication in the presence of replication-inhibitory DNA lesions, thereby allowing the repair of such lesions prior to completion of DNA replication, the simultaneous sequestration of RPA from such repair pathways would potentially abrogate any advantage of replication arrest. The answer to this question must await a greater understanding of the nature and regulation of the NCL-RPA interaction and/or direct assays of RPAdependent DNA repair pathways in the presence of interacting NCL-RPA. However, it should be noted that RPA proteins undergo extensive post-translational modification to regulate the differing activities of this complex (Prakash and Borgstahl 2012); indeed, CDK2/PI3K family-targeted phosphosites have been identified on RPA2 that are able to drive RPA's partitioning into DNA damage repair pathways at the expense of DNA replication (Vassin et al. 2004). Such modifications represent potential regulatory nodes 
that may separate the inhibitory effects of NCL-RPA on DNA replication from unintended ones on DNA repair pathways.

\section{Partners in crime(s): NPM1 coordinates APE1's roles in BER and rRNA quality control}

Base excision repair (BER) represents the major cellular response to a wide range of genotoxic stresses that result in damage or removal of helix-embedded DNA bases or nucleotides without causing distortion of the DNA helix; such stresses include oxidative damage, DNA alkylating agents such as methyl methanesulfonate (MMS) and other base modifiers such as cisplatin (Carter and Parsons 2016). BER can occur in two forms, 'short-patch' or 'long-patch'. Short-patch BER uses a series of steps to: i) excise the damaged base from the DNA, leaving an apurinic/apyrimidinic (AP) site (catalyzed by one of several DNA glycosylases); ii) endonucleolytically cleave at the AP site, leaving a one-base DNA gap (AP endonuclease 1, APE1); iii) fill the gap with a complementary base pair (DNA Pol $\beta$ ); and iv) religate the remaining DNA nick (DNA ligase III/XRCC1 complex). While long-patch BER is initiated by the same mechanism, in this case the DNA Pol $\beta$ hands off to replicative DNA Pol $\delta / \varepsilon$ which adds a short 2-8 nt run to the repaired site, creating a short displaced DNA 'flap' which is excised by flap endonuclease 1 (FEN1) and religated by DNA ligase I in PCNA-dependent reactions (Carter and Parsons 2016). The complexity of this repair system and its ubiquitous activity - cells are estimated to generate $>10,000$ DNA base lesions per day - make it an important regulatory target (Lindahl 1993).

The first evidence for an involvement of NPM1 in the regulation of BER came from proteomic studies that identified NPM1 as a major interactor of APE1 in unstressed cells (Vascotto et al. 2009). This interaction, mediated by binding of the lysine-rich APE1 N-terminal tail to NPM1's N-terminal region containing the oligomerization domain and acidic tracts, results in the robust recruitment of APE1 into nucleoli (Fantini et al. 2010; Vascotto et al. 2009). Upon induction of genotoxic stress such as base 
oxidation or methylation, lysines within the APE1 N-terminal become acetylated, interrupting the NPM1-APE1 interactions and inducing translocation of APE1 from the nucleolus to the nucleoplasm (Fantini et al. 2010; Lirussi et al. 2012). Mutation of four of these residues (K27, K31, K32, K35) to acetylation-mimicking uncharged alanines (termed $A P E 1^{\text {K4pleA }}$ ) results in constitutive translocation of APE1 to the nucleoplasm, while inhibition of acetylation by lysine-to-arginine mutations (APE1 ${ }^{\text {KApleR }}$ ) constitutively prevents translocation; these same mutations inhibit (APE1 ${ }^{\text {K4pleA }}$ ) or enhance (APE1 ${ }^{\text {K4pleR }}$ ) the interaction of APE1 with NPM1, emphasizing the importance of this interaction to the regulation of APE1's nucleolar sequestration (Lirussi et al. 2012). Notably, the APE1 ${ }^{\text {K4pleA }}$ mutation shows significantly greater endonuclease activity on AP-DNA and its expression in vivo reduces AP site accumulation, suggesting that sequestration into the nucleolus by NPM1 prevents APE1 action within the BER pathway (Lirussi et al. 2012). Several other BER proteins including FEN1 and Ligase III are also reported to exhibit NPM1-dependent nucleolar localization, which may be reversed following genotoxic stress, suggesting that this mechanism may control multiple steps of the BER repair pathway (Poletto et al. 2014).

While this model seems, on the surface, a straightforward analog of the NPM1-p19ARF sequestration model described earlier, several observations have suggested that the regulation may be more complicated. The first is that, while APE1 is enriched in the nucleoli, extensive nucleoplasmic APE1 staining is still observed in most cells, suggesting that NPM1 sequesters only a relatively minor pool (an estimated 5-10\% of total cellular APE1) into nucleoli (Lirussi et al. 2012; Vascotto et al. 2009). Furthermore, while induction of genotoxic stress is reported to reduce the interaction of NPM1-APE1 in the nucleolus and release APE1 to the nucleoplasm, proximity ligation assays suggest that NPM1 and APE1 may in fact interact in the nucleoplasm as well as the nucleolus, with NPM1 possibly modulating APE1 activity in the nucleoplasm (Vascotto et al. 2014). Thirdly, under the model outlined above, NPM1 is expected to act as a negative regulator of APE1. However, studies have instead observed that, in vitro, NPM1 binding to APE1 enhances its AP-endonuclease activity (Vascotto et al. 2009) and that, in vivo, 
APE1 shows reduced AP-endonuclease activity in $\mathrm{NPM1}^{-/} \mathrm{p} 53^{-/}$cell lysates compared to $p 53^{--} \mathrm{NPM}^{+/+}$ lysates, despite no longer being sequestered in nucleoli (Vascotto et al. 2014). NPM1-- cells also showed an increased sensitivity to $\mathrm{BER}$-inducing agents such as $\mathrm{H}_{2} \mathrm{O}_{2}, \mathrm{KBrO}_{3}$ and $\mathrm{MMS}$, and carried a greater number of AP-sites compared to $\mathrm{NPM1}^{+/+}$cells, suggesting that NPM1 is in fact required for APE1 function in vivo (Vascotto et al. 2014). Finally, an ongoing argument concerns the role of SIRT1, a lysine deacetylase, in the APE1-NPM1 regulatory axis. An initial report found that a SIRT1-APE1 interaction was enhanced by genotoxic stress, and that this interaction drove deacetylation of APE1 at K6/K7. Furthermore, they reported that SIRT1 activity mediated the increased association of APE1 with XRCC1, a key BER scaffolding enzyme, in response to MMS treatment, and that depletion of SIRT1 resulted in sensitivity of HeLa cells to MMS and increased AP-site accumulation, both of which could be rescued by overexpression of APE1 (Yamamori et al. 2010). However, subsequent work found that SIRT1 interacted with $A P E 1^{\text {K4pleR }}$ but not with the acetylation-mimicking $A P E 1^{\text {K4pleA }}$ mutant that is released from the nucleolus by NPM1 upon induction of genotoxic stress, and failed to deacetylate APE1 ${ }^{\mathrm{K} p l e A}$ at K6/K7, despite the fact that this form possesses greater AP-endonuclease activity than either APE1 ${ }^{\text {WT }}$ or APE1 ${ }^{\text {K4pleR }}$ (Lirussi et al. 2012).

The explanation of these functional disagreements will require considerable further work on the nature and regulation of the NPM1-APE1 interaction; however, several points can be raised that may provide some insight. The observation that NPM1 is able to mediate the nucleolar localization and expression of other BER proteins including FEN1 and Ligase III (Poletto et al. 2014) and that it is able to control the transcription of BER genes through its interaction with transcription factors such as p53 and pRb/E2F1 (Lin et al. 2010) may explain the sensitization of $N P M^{-/}$cells to genotoxic damage independently of any positive-regulatory role on APE1. Alternatively, the sequestration of APE1 into the nucleolus by NPM1 may be required for its full activation in vivo, for example through promoting post-translational modification by particular enzymes, which may be restricted to nucleoli. Interestingly, APE1 has been 
reported to undergo HDM2-dependent ubiquitination on its $\mathrm{N}$-terminal lysine residues, including the K27 residue targeted in the $A P E 1^{\text {K4pleA }}$ mutant (Busso et al. 2009); such a modification could significantly alter the activity, stability and/or binding of APE1 to partner proteins. This modification is of particular interest given the recent report of a ubiquitin-binding domain in NPM1 (Koike et al. 2010), which could, hypothetically, mediate alternative binding modes of NPM1 to APE1-Ub. With regards to SIRT1 involvement in the regulation of APE1, it is important to note that there is no available data on whether NPM1 and SIRT1 are able to modulate each other's activity on APE1, nor the identity and regulation of the acetylase(s) responsible for APE1 acetylation at K6/K7 and/or K27/K31/K32/K35. Such experiments will be particularly valuable in dissecting the role of SIRT1 and NPM1 in the relative regulation of APE1'S activity in vivo. Interestingly, experiments by Lirussi et al. (2012) did observe a deacetylation of APE1 at $\mathrm{K} 27 / \mathrm{K} 31 / \mathrm{K} 32 / \mathrm{K} 35$ as well as at K6/K7 by SIRT1, suggesting that SIRT1 may be able to modulate the interaction of NPM1 with APE1 directly, as well as potentially promote its own interaction with APE1 through a two-step process involving K27/K31/K32/K35 deacetylation followed by binding and K6/K7 regulation.

A final, significant consideration in the regulation of APE1 by NPM1 concerns roles for APE1 outside of the BER pathway. Like NPM1 and NCL, APE1 is a remarkably pleiotropic protein that, in addition to possessing activity within BER, is able to independently regulate cellular redox sensing and act as a coactivator for several transcription factors (Antoniali et al. 2014). Strikingly, APE1's AP-endonuclease activity has been shown to be active not only on cellular DNA but to mediate endonucleolytic cleavage of AP-containing SSRNA (Berquist et al. 2008); in vivo, depletion of APE1 results in increased oxidation and AP-accumulation in ribosomal RNA and, consequently, decreased protein synthesis and cellular proliferation. These observations demonstrate that APE1 regulates a previously unappreciated 'quality control' mechanism for nucleolar rRNA (Vascotto et al. 2009). The observation that SMUG1 DNA glycosylase is also able to metabolize base-oxidized rRNA within the nucleolus (Jobert et al. 2013), and 
that several other BER proteins are targeted to the nucleolus via NPM1 (Poletto et al. 2014) and may regulate rRNA transcription and/or repair (Aas et al. 2003), suggest that NPM1 may be responsible for the redeployment of canonical BER proteins into a novel nucleolar rRNA quality control pathway. Importantly, depletion of NPM1 is likely to compromise this pathway, suggesting that the observed sensitivity of $\mathrm{NPM}^{-/}$cells to oxidizing/alkylating agents may arise from their activity on rRNA rather than DNA. This observation further raises the possibility that NPM1-mediated sequestration to the nucleolus may in fact not be a DNA repair regulatory pathway at all, but instead a means by which NPM1 may coordinate degradation or repair of oxidatively damaged rRNA.

\section{Fix it or fudge it: Alternative responses to UV-induced damage controlled by NCL and NPM1}

While BER is responsible for the repair of small, single-base lesions that are capable of being excised by DNA glycosylases and/or APE1, there are numerous forms of single-strand DNA damage that are refractory to this pathway including bulky base adducts, products of UV irradiation such as cyclobutanepyrimidine dimers (CPDs) and 6,4-pyrimidine-pyrimidone photoproducts (6,4-PPs), and intrastrand crosslinks generated by agents such as cisplatin, among others. These lesions are all repaired by the nucleotide excision repair (NER) pathway. Two forms of NER exist, global genomic (GG)-NER, which recognizes lesions occurring throughout the genome, and transcription-coupled (TC)-NER, which recognizes lesions causing arrest of RNA Polymerase II during transcriptional elongation (Marteijn et al. 2014). Although the initial DNA damage recognition factors for GG- and TC-NER vary depending on the recognizing pathway and the type of lesion involved, all converge on the assembly of the TFIIH complex at the site of damage, and unwinding of DNA in an extended region around the damage site; this extended section of ssDNA is stabilized by interaction with RPA. The ssDNA strand containing the lesion is excised at both ends by XPF and XPG, and the PCNA replicative helicase recruits DNA polymerases $\delta, \varepsilon$ 
or $\mathrm{k}$, one or more of which fills the ssDNA gap prior to ligation of the repaired DNA (Marteijn et al. 2014).

Unlike for BER and DSB repair, evidence for NER-regulatory roles of NPM1 and NCL is sparse. Phenotypic analyses have revealed increased resistance to UV-induced DNA damage following NCL kd (Yang et al. 2009) or NPM1 overexpression (Wu et al. 2002a), though measurements of UV radiosensitivity following NPM1 kd/deletion have yielded conflicting results (Vascotto et al. 2014; Wu et al. 2002b). Separate groups have also used a biochemical assay for repair of a UV-irradiated reporter plasmid to demonstrate decreased NER activity following either overexpression of NCL (Yang et al. 2009) or depletion of NPM1 (Wu et al. 2002b), suggesting that these proteins may have opposing activities in NER regulation. It is important to note, however, that neither of these experimental approaches are able to differentiate between direct activities of NPM1/NCL at sites of NER and global NER deficiencies arising indirectly, such as from NPM1/NCL-dependent transcriptional modulation of the NER machinery (Lin et al. 2010; Liu et al. 2007; Wu et al. 2002a; Wu et al. 2002b). Indeed, Wu et al. (2002a, 2002b) observed that expression levels of PCNA were closely correlated with those of NPM1 in their system, likely explaining the observed effects on NER efficiency. Yang et al. (2009) did report a direct interaction between NCL and PCNA that was enhanced following UV irradiation, and found that overexpression of PCNA was able to rescue NCL overexpression-induced NER defects. However, it was not demonstrated that the NCL-PCNA interaction itself was reponsible for NCL's modulation of NER, leaving open the possibility that NCL may regulate NER indirectly through a pathway dependent on PCNA - for example, through transcriptional and/or post-transcriptional modulation of PCNA, or through regulation of other PCNA-regulatory NER components such as RPA (Daniely and Borowiec 2000; Kim et al. 2005). Considerable further work will be required to establish the precise mechanistic roles, if any, of NPM1 and/or NCL in NER.

While the NER pathway is able to repair the majority of bulky single-strand DNA lesions occurring in the cell, its reliance on strand excision and gap filling renders it unable to repair unpaired DNA lesions such 
as those that fall in a regions of ssDNA or are discovered by a progressing replication fork. In these cases, an alternative response, termed Translesion Synthesis (TLS), is employed in which the cell recruits one of a number of DNA polymerases (Rev1 or Pols $k, \eta, \zeta$ or $\mathrm{l}$ ) that are specifically able to introduce a nucleotide opposite the site of DNA damage and thus replicate past the lesion, albeit at the expense of an increased rate of replicational mutagenesis arising from the more mismatch/error-permissive nature of these polymerases (Waters et al. 2009). As for NER, different TLS polymerases possess specificity for different DNA lesions, with DNA Pol $\eta$ being predominantly responsible for the repair of UV-induced lesions (Waters et al. 2009).

Recently, a paper from Ziv et al. (2014) has revealed that, instead of regulating NER-mediated repair of UV-induced DNA lesions, NPM1 may instead promote their bypass via TLS. Using a detailed screening process to identify genes required for TLS following UV irradiation, this study found that siRNA depletion of NPM1 resulted in a global TLS defect across all DNA lesions tested, in particular UV-induced CPDs and 6,4-PPs. Consistent with these observations, NPM1 was able to interact directly with Pol $\eta$ in a manner that both prevented proteasomal degradation of Pol $\eta$ and sequestered Pol $\eta$ away from replication foci, maintaining a cellular stockpile of Pol $\eta$ in an inactive state. Upon UV irradiation, the interaction between NPM1 and Pol $\eta$ was abolished, allowing Pol $\eta$ to move to replication foci and perform TLS past newly-formed CPDs/6,4-PPs (Ziv et al. 2014). While these observations are so far incomplete, they provide an important mechanism by which NPM1 may be directly able to promote cellular resistance to, and recovery from, UV irradiation.

\section{Discussion}

In this review we have explored the roles played by two abundant nucleolar proteins, nucleophosmin (NPM1) and nucleolin (NCL), in the numerous DNA damage pathways existent in mammalian cells. 
Remarkably, regulatory roles have been found for both of these ribosome biogenesis factors in the processes of NHEJ, HR, BER, NER, TLS and DNA replication inhibition (see Fig. 2). It is important to note that the roles reviewed are mediated by direct contact between NPM1/NCL and components of the discussed repair machineries; these proteins may promote numerous other, indirect effects on DNA damage repair, cell proliferation and apoptosis through their ability to regulate the transcription, translation and/or stability of numerous important cellular factors including p53 and c-Myc, along with many others (Colombo et al. 2011; Tajrishi et al. 2011). The pleiotropic functions of NCL and NPM1 in DDR, along with their established roles in processes as diverse as ribosome biogenesis, transcription initiation, mRNA maturation and centrosome maturation, establish the credentials of these proteins as regulatory hubs in the coordination of cellular growth, repair and proliferation and emphasize their potential as targets for multivalent chemotherapeutic agents (Colombo et al. 2011; Tajrishi et al. 2011).

\section{Guiding principles of NPM1/NCL in DDR: chaperoning and sequestration}

While considerable further research is required to fully describe the mechanism(s) by which NCL and NPM1 are able to regulate different DNA repair pathways, several interesting concepts are beginning to emerge. The histone chaperone activities of NCL and NPM1 have been known for at least 10 years (Angelov et al. 2006; Okuwaki et al. 2001), as have their ability to regulate activity on Pol Il-transcribed loci (Angelov et al. 2006). However, the widespread importance of these activities are only now becoming clear with the observations of their contribution to diverse processes including rDNA repeat silencing (Cong et al. 2012), centrosome stability (Foltz et al. 2009) and, most recently, chromatin remodeling during HR and/or NHEJ (Goldstein et al. 2013; Kobayashi et al. 2012). These observations suggest that histone chaperoning may be a common mechanism to many of NPM1 and NCL's functions; given that chromatin remodeling is known to be a major factor in the activities of all DNA repair 
pathways it is possible that histone chaperoning by NCL and NPM1 may in the future be recognized as regulatory activities contributing to their role(s) in other DDR and non-DDR pathways (Dinant et al. 2012; Hinz and Czaja 2015; Jeggo and Downs 2014; Li 2014).

A similarly emergent concept surrounds the ubiquity of sequestration mechanisms in the regulatory activities of NCL/NPM1, and their cause. This review has demonstrated that both NCL and NPM1 regulate multiple DDR pathways through the sequestration of key proteins in inactive states (RPA/PCNA and APE1/Pol $\eta$, respectively). Indeed, the eviction of histone dimers discussed above could be considered a particular application of this sequestration activity, preventing mature histones from forming damage site-proximal nucleosomes until completion of DDR - a model analogous to that recently reported for NPM2 during embryogenesis (Onikubo et al. 2015). Sequestration-based systems are particularly advantageous to the cell in that they can be rapidly deployed without nascent protein synthesis, can be tightly controlled and titrated by regulatory partners and/or post-translational modifications, and can be reversed upon resolution of DNA insult or removal of stress. It is also significant that NCL and NPM1 represent ideal candidates as potential 'molecular sponges' for interacting proteins due to their high abundance, diversity of interacting domains/mechanisms and, in the case of NPM1, its ability to oligomerize and form large, multi-subunit complexes capable of sequestering multiple binding partners in large, non-functional aggregates. However, caution should be applied when considering the implications of DDR protein-NPM1/NCL interactions. While sequestration may represent a common mechanism by which NPM1 and NCL control DNA repair dynamics, these interactions can have consequences for the activities of NPM1/NCL themselves, as demonstrated by role of p53 in the regulation of NCL nucleolar translocation (Daniely et al. 2002). Additionally, the discovery of DDR-independent roles for APE1 and other NPM1-binding BER factors in nucleolar rRNA quality control (Berquist et al. 2008; Guo et al. 2008; Poletto et al. 2014; Vascotto et al. 2009) suggest that 
NPM1 and/or NCL may go beyond being passive 'sponges' for such proteins and may play a key role in the coordination of their diverse cellular activities.

Nucleoplasmic translocation: a mechanism of recruitment, or a means of exclusion?

A growing point of contention concerns the contribution of nucleoplasmic translocation of NCL and NPM1 to their activity. While at first glance the different genotoxic conditions that induce nucleoplasmic translocation of these two proteins correlate well with their respective DNA repair activities, and the translocation correlates temporally and spatially with the functions of their partner proteins, several inconsistencies are beginning to emerge. Nucleoplasmic translocation of $\mathrm{NCL}$ was found to require binding of NCL to p53 (Daniely et al. 2002); however, the NCL-RPA interaction, reported to be exclusively nucleoplasmic, occurred in the absence of p53, suggesting either that the translocation required for this interaction bypasses $\mathrm{p} 53$, or that the $\mathrm{NCL}$ involved is part of a pre-existing nucleoplasmic pool (Kim et al. 2005). Similarly, NPM1 has been reported not to undergo nucleoplasmic translocation in response to IR treatment, but was nevertheless found within DSB repair foci in cells treated with IR (Koike et al. 2010; Lee et al. 2005). In light of these observations, several hypotheses can be proposed. NCL and NPM1 are both proteins that carry a high number of post-translational modifications, and evidence continues to accumulate suggesting that the nucleoplasmic translocation of both NCL (Kim et al. 2005; Zhang et al. 2015) and NPM1 (Liu et al. 2006; Liu et al. 2007; Sato et al. 2004; Yogev et al. 2008) may be regulated by multiple independent modifications and pathways in response to different genotoxic stresses or in distinct cell types. Moreover, the fact that NPM1 localization to DSBs could only be detected using an antibody recognizing the phosphorylated NPM1-pT199 suggests that any stress-induced relocalization of NPM1, and potentially NCL, may involve a small and specifically-modified population of these highly abundant proteins, and that such fractional relocalization may be unobservable with the currently 
available assays. A final and intriguing possibility is that the nucleoplasmic translocation of NCL and/or NPM1 may represent not so much a mechanism for the enhancement of DNA repair, but rather one for the inhibition of rRNA synthesis and processing. Both NCL and NPM1 are recruited to rDNA genes and directly regulate its transcription, as well as a number of post-transcriptional steps of pre-rRNA processing. While several pathways have already been identified by which the DNA damage response can induce inhibition of rDNA transcription (Boulon et al. 2010), the nucleoplasmic translocation of NCL and NPM1 may represent an important mechanism to reinforce and maintain this inhibition until the DNA insult is resolved. Additionally, such translocation may also represent a previously unappreciated method by which the post-transcriptional processing of pre-rRNA may be inhibited following DNA damage.

Ultimately, the data discussed in this review represent only the initial steps in characterizing the roles of NPM1 and NCL in the clearance of diverse DNA damages in vivo. However, what has been thus far described is sufficient to underline the remarkable multifunctionality of these proteins across multiple independent DDR pathways, as well as numerous other processes essential to cellular growth and proliferation including transcription, stress sensing and ribosome biogenesis. Given this pleiotropy, it is not surprising that perturbations of these factors have been linked to the development of numerous cancers and myeloproliferative disorders. Mutations in the NPM1 gene are particularly common among myeloid cancers, and are the single most common genetic cause of acute myeloid leukemia. Particularly frequent among these are mutations in the NPM1 NLSs, which cause aberrant cytoplasmic accumulation of NPM1 (termed NPM1c+) and associated DNA repair factors, marking NPM1 as a key tumor suppressor in myeloid lineages (Federici and Falini 2013). Conversely, other studies have found NPM1 overexpression to be a common feature of solid tumors from numerous different tissues, demonstrating that NPM1 can act as either a tumor suppressor or an oncogene depending on the cell and/or tissue type affected (Colombo et al. 2011). Tumor suppressor activities have not been reported for NCL so far; 
however, overexpression of NCL has been linked to the development, progression and malignancy of a wide variety of solid and hematological tumors, identifying this protein as a major oncogene in numerous cellular systems (Berger et al. 2015). This diversity of pathogenic roles for NPM1 and NCL, and their contribution to numerous essential proliferative pathways, make these two proteins exciting future targets for the development of multi-active chemotherapeutic agents (Poletto et al. 2015; Sekhar et al. 2011).

\section{Acknowledgements}

The authors thank Dr. C. Trahan, L. C. Aguilar and M. Y. Delubac for critical reading of the manuscript. This work was supported by funding from the Natural Sciences and Engineering Research Council of Canada (RGPIN 386315-MO). M.O. holds a CIHR New Investigator award. 


\section{References}

Aas, P.A., Otterlei, M., Falnes, P.O., Vagbo, C.B., Skorpen, F., Akbari, M., Sundheim, O., Bjoras, M., Slupphaug, G., Seeberg, E., and Krokan, H.E. 2003. Human and bacterial oxidative demethylases repair alkylation damage in both RNA and DNA. Nature 421(6925): 859-863. doi: 10.1038/nature01363. Ahmad, Y., Boisvert, F.M., Gregor, P., Cobley, A., and Lamond, A.I. 2009. NOPdb: Nucleolar proteome database--2008 update. Nucleic Acids Research 37(Database issue): 4. doi: 10.1093/nar/gkn804. Andersen, J.S., Lam, Y.W., Leung, A.K.L., Ong, S.-E., Lyon, C.E., Lamond, A.I., and Mann, M. 2005. Nucleolar proteome dynamics. Nature 433(7021): 77-83. doi: 10.1038/nature03207 Andersen, J.S., Lyon, C.E., Fox, A.H., Leung, A.K.L., Lam, Y.W., Steen, H., Mann, M., and Lamond, A.I. 2002. Directed proteomic analysis of the human nucleolus. Current Biology 12(1): 1-11. doi: 10.1016/S0960-9822(01)00650-9.

Angelov, D., Bondarenko, V.A., Almagro, S., Menoni, H., Mongélard, F., Hans, F., Mietton, F., Studitsky, V.M., Hamiche, A., Dimitrov, S., and Bouvet, P. 2006. Nucleolin is a histone chaperone with FACT-like activity and assists remodeling of nucleosomes. EMBO Journal 25(8): 1669-1679. doi: 10.1038/sj.emboj.7601046.

Antoniali, G., Lirussi, L., Poletto, M., and Tell, G. 2014. Emerging roles of the nucleolus in regulating the DNA damage response: the noncanonical DNA repair enzyme APE1/Ref-1 as a paradigmatical example. Antioxidants and Redox Signaling 20(4): 621-639. doi: 10.1089/ars.2013.5491.

Bao, Y. 2011. Chromatin response to DNA double-strand break damage. Epigenomics 3(3): 307-321. doi: 10.2217/epi.11.14.

Battu, A., Ray, A., and Wani, A.A. 2011. ASF1A and ATM regulate H3K56-mediated cell-cycle checkpoint recovery in response to UV irradiation. Nucleic Acids Research 39(18): 7931-7945. doi:

10.1093/nar/gkr523. 
Berger, C.M., Gaume, X., and Bouvet, P. 2015. The roles of nucleolin subcellular localization in cancer. Biochimie 113: 78-85. doi: 10.1016/j.biochi.2015.03.023.

Berquist, B.R., McNeill, D.R., and Wilson, D.M., 3rd. 2008. Characterization of abasic endonuclease activity of human Ape1 on alternative substrates, as well as effects of ATP and sequence context on AP site incision. Journal of Molecular Biology 379(1): 17-27. doi: 10.1016/j.jmb.2008.03.053.

Bhatt, P., d'Avout, C., Kane, N.S., Borowiec, J.A., and Saxena, A. 2012. Specific domains of nucleolin interact with Hdm2 and antagonize Hdm2-mediated p53 ubiquitination. FEBS Journal 279(3): 370-383. doi: 10.1111/j.1742-4658.2011.08430.x.

Bieging, K.T., Mello, S.S., and Attardi, L.D. 2014. Unravelling mechanisms of p53-mediated tumour suppression. Nature Reviews: Cancer 14(5): 359-370. doi: 10.1038/nrc3711.

Boisvert, F.M., Lam, Y.W., Lamont, D., and Lamond, A.I. 2010. A quantitative proteomics analysis of subcellular proteome localization and changes induced by DNA damage. Molecular and Cellular Proteomics 9(3): 457-470. doi: 10.1074/mcp.M900429-MCP200.

Boisvert, F.M., van Koningsbruggen, S., Navascues, J., and Lamond, A.I. 2007. The multifunctional nucleolus. Nature Review Molecular Cell Biology 8(7): 574-585. doi: 10.1038/nrm2184.

Borer, R.A., Lehner, C.F., Eppenberger, H.M., and Nigg, E.A. 1989. Major nucleolar proteins shuttle between nucleus and cytoplasm. Cell 56(3): 379-390. doi: 10.1016/0092-8674(89)90241-9.

Borggrefe, T., Wabl, M., Akhmedov, A.T., and Jessberger, R. 1998. A B-cell-specific DNA recombination complex. Journal of Biological Chemistry 273(27): 17025-17035. doi: 10.1074/jbc.273.27.17025.

Boulon, S., Westman, B.J., Hutten, S., Boisvert, F.-M., and Lamond, A.I. 2010. The nucleolus under stress. Molecular Cell 40(2): 216-227. doi: 10.1016/j.molcel.2010.09.024.

Busso, C.S., Iwakuma, T., and Izumi, T. 2009. Ubiquitination of mammalian AP endonuclease (APE1) regulated by the p53-MDM2 signaling pathway. Oncogene 28(13): 1616-1625. doi: 10.1038/onc.2009.5. 
Carter, R.J., and Parsons, J.L. 2016. Base excision repair: A pathway regulated by post-translational modifications. Molecular and Cellular Biology in press. doi: 10.1128/mcb.00030-16.

Chen, C.-C., Carson, J.J., Feser, J., Tamburini, B., Zabaronick, S., Linger, J., and Tyler, J.K. 2008. Acetylated lysine 56 on histone H3 drives chromatin assembly after repair and signals for the completion of repair.

Cell 134(2): 231-243. doi: 10.1016/j.cell.2008.06.035.

Chen, C. M.; Chiang, S. Y. and Yeh, N. H. 1991. Increased stability of nucleolin in proliferating cells by inhibition of its self-cleaving activity. Journal of Biological Chemistry 266(12): 7754-7758.

Colombo, E., Alcalay, M., and Pelicci, P.G. 2011. Nucleophosmin and its complex network: a possible therapeutic target in hematological diseases. Oncogene 30: 2595-2609. doi: 10.1038/onc.2010.646 Colombo, E., Marine, J.-C., Danovi, D., Falini, B., and Pelicci, P.G. 2002. Nucleophosmin regulates the stability and transcriptional activity of p53. Nature Cell Biology 4(7): 529-533. doi: 10.1038/ncb814 Cong, R., Das, S., Ugrinova, I., Kumar, S., Mongelard, F., Wong, J., and Bouvet, P. 2012. Interaction of nucleolin with ribosomal RNA genes and its role in RNA polymerase I transcription. Nucleic Acids Research 40(19): 9441-9454. doi: 10.1093/nar/gks720.

Daniely, Y., and Borowiec, J.A. 2000. Formation of a complex between nucleolin and replication protein A after cell stress prevents initiation of DNA replication. Journal of Cell Biology 149(4): 799-810. doi: 10.1083/jcb.149.4.799.

Daniely, Y., Dimitrova, D.D., and Borowiec, J.A. 2002. Stress-dependent nucleolin mobilization mediated by p53-nucleolin complex formation. Molecular and Cellular Biology 22(16): 6014-6022. doi:

10.1128/MCB.22.16.6014-6022.2002.

De, A., Donahue, S.L., Tabah, A., Castro, N.E., Mraz, N., Cruise, J.L., and Campbell, C. 2006. A novel interaction of nucleolin with Rad51. Biochemical and Biophysical Research Communications 344(1): 206213. doi: 10.1016/j.bbrc.2006.03.113. 
Dempsey, L.A., Hanakahi, L.A., and Maizels, N. 1998. A specific isoform of hnRNP D interacts with DNA in the LR1 heterodimer: canonical RNA binding motifs in a sequence-specific duplex DNA binding protein. Journal of Biological Chemistry 273(44): 29224-29229. doi: 10.1074/jbc.273.44.29224.

Dinant, C., Bartek, J., and Bekker-Jensen, S. 2012. Histone displacement during nucleotide excision repair. International Journal of Molecular Science 13(10): 13322-13337. doi: 10.3390/ijms131013322.

Durut, N., and Saez-Vasquez, J. 2015. Nucleolin: dual roles in rDNA chromatin transcription. Gene 556(1): 7-12. doi: 10.1016/j.gene.2014.09.023.

Dutta, S., Akey, I.V., Dingwall, C., Hartman, K.L., Laue, T., Nolte, R.T., Head, J.F., and Akey, C.W. 2001. The crystal structure of nucleoplasmin-core: implications for histone binding and nucleosome assembly. Mol Cell 8(4): 841-853. doi: 10.1016/S1097-2765(01)00354-9.

Edlich-Muth, C., Artero, J.-B., Callow, P., Przewloka, M.R., Watson, A.A., Zhang, W., Glover, D.M., Debski, J., Dadlez, M., Round, A.R., Forsyth, V.T., and Laue, E.D. 2015. The pentameric nucleoplasmin fold is present in Drosophila FKBP39 and a large number of chromatin-related proteins. J Mol Biol 427(10): 1949-1963. doi: 10.1016/j.jmb.2015.03.010.

Emmott, E., and Hiscox, J.A. 2009. Nucleolar targeting: the hub of the matter. EMBO Reports 10(3): 231238. doi: 10.1038/embor.2009.14.

Fang, S.-H. and Yeh, N.-H. 1993. The self-cleaving activity of nucleolin determines its molecular dynamics in relation to cell proliferation. Experimental Cell Research 208(1): 48-53. doi: 10.1006/excr.1993.1221. Fantini, D., Vascotto, C., Marasco, D., D’Ambrosio, C., Romanello, M., Vitagliano, L., Pedone, C., Poletto, M., Cesaratto, L., Quadrifoglio, F., Scaloni, A., Radicella, J.P., and Tell, G. 2010. Critical lysine residues within the overlooked N-terminal domain of human APE1 regulate its biological functions. Nucleic Acids Research 38(22): 8239-8256. doi: 10.1093/nar/gkq691.

Federici, L., and Falini, B. 2013. Nucleophosmin mutations in acute myeloid leukemia: a tale of protein unfolding and mislocalization. Protein Science 22(5): 545-556. doi: 10.1002/pro.2240. 
Fishel, R. 2015. Mismatch repair. Journal of Biological Chemistry 290(44): 26395-26403. doi:

10.1074/jbc.R115.660142.

Finn, R.M., Ellard, K., Eirín-López, J.M., and Ausió, J. 2012. Vertebrate nucleoplasmin and NASP: egg histone storage proteins with multiple chaperone activities. FASEB J 26(12): 4788-4804. doi:

10.1096/fj.12-216663.

Foltz, D.R., Jansen, L.E.T., Bailey, A.O., Yates, J.R., III, Bassett, E.A., Wood, S., Black, B.E., and Cleveland, D.W. 2009. Centromere-specific assembly of CENP-A nucleosomes is mediated by HJURP. Cell 137(3): 472-484. doi: 10.1016/j.cell.2009.02.039.

Franco, G., Bañuelos, S., Falces, J., Muga, A., and Urbaneja, M.A. 2008. Thermodynamic characterization of nucleoplasmin unfolding: interplay between function and stability. Biochemistry 47(30): 7954-7962. doi: $10.1021 /$ bi8002555.

Fujiki, H., Watanabe, T., and Suganuma, M. 2014. Cell-surface nucleolin acts as a central mediator for carcinogenic, anti-carcinogenic, and disease-related ligands. Journal of Cancer Research and Clinical Oncology 140(5): 689-699. doi: 10.1007/s00432-014-1587-5.

Ginisty, H., Serin, G., Ghisolfi-Nieto, L., Roger, B., Libante, V., Amalric, F., and Bouvet, P. 2000. Interaction of nucleolin with an evolutionarily conserved pre-ribosomal RNA sequence is required for the assembly of the primary processing complex. Journal of Biological Chemistry 275(25): 18845-18850. doi:

10.1074/jbc.M002350200.

Ginisty, H., Sicard, H., Roger, B., and Bouvet, P. 1999. Structure and functions of nucleolin. Journal of Cell Science 112 (6): 761-772.

Gjerset, R.A. 2006. DNA damage, p14ARF, nucleophosmin (NPM/B23), and cancer. Journal of Molecular Histology 37(5-7): 239-251. doi: 10.1007/s10735-006-9040-y. 
Goldstein, M., Derheimer, F.A., Tait-Mulder, J., and Kastan, M.B. 2013. Nucleolin mediates nucleosome disruption critical for DNA double-strand break repair. Proceedings of the National Academy of Sciences of the United States of America 110(42): 16874-16879. doi: 10.1073/pnas.1306160110.

González, V., Guo, K., Hurley, L., and Sun, D. 2009. Identification and characterization of nucleolin as a c-myc G-quadruplex-binding protein. Journal of Biological Chemistry 284(35): 23622-23635. doi:

10.1074/jbc.M109.018028.

González, V., and Hurley, L.H. 2010. The C-terminus of nucleolin promotes the formation of the c-MYC G-quadruplex and inhibits c-MYC promoter activity. Biochemistry 49(45): 9706-9714. doi:

10.1021/bi100509s.

Grisendi, S., Bernardi, R., Rossi, M., Cheng, K., Khandker, L., Manova, K., and Pandolfi, P.P. 2005. Role of nucleophosmin in embryonic development and tumorigenesis. Nature 437(7055): 147-153. doi:

10.1038/nature03915.

Gudavicius, G., Dilworth, D., Serpa, J.J., Sessler, N., Petrotchenko, E.V., Borchers, C.H., and Nelson, C.J. 2014. The prolyl isomerase, FKBP25, interacts with RNA-engaged nucleolin and the pre-60S ribosomal subunit. RNA 20(7): 1014-1022. doi: 10.1261/rna.042648.113.

Gurumurthy, M.; Tan, C.H.; Ng, R.; Zeiger, L.; Lau, J.; Lee, J.; Dey, A.; Philp, R.; Li, Q.; Lim, T.M.; Price, D.H.; Lane, D.P. and Chao, S.H. 2008. Nucleophosmin interacts with HEXIM1 and regulates RNA polymerase II transcription. Journal of Molecular Biology 378(2): 302-317. doi: 10.1016/j.jmb.2008.02.055.

Hanakahi, L.A., Bu, Z., and Maizels, N. 2000. The C-terminal domain of nucleolin accelerates nucleic acid annealing. Biochemistry 39(50): 15493-15499. doi: 10.1021/bi001683y.

Hanakahi, L.A., Dempsey, L.A., Li, M.-J., and Maizels, N. 1997. Nucleolin is one component of the B cellspecific transcription factor and switch region binding protein, LR1. Proceedings of the National Academy of Sciences of the United States of America 94(8): 3605-3610. 
Hanakahi, L.A., Sun, H., and Maizels, N. 1999. High affinity interactions of nucleolin with G-G-paired rDNA. Journal of Biological Chemistry 274(22): 15908-15912. doi: 10.1074/jbc.274.22.15908. Henras, A.K., Plisson-Chastang, C., O'Donohue, M.F., Chakraborty, A., and Gleizes, P.E. 2014. An overview of pre-ribosomal RNA processing in eukaryotes. Wiley Interdisciplinary Reviews: RNA 6(2): 225242. doi: 10.1002/wrna.1269.

Hernandez-Verdun, D., Roussel, P., Thiry, M., Sirri, V., and Lafontaine, D.L.J. 2010. The nucleolus: structure/function relationship in RNA metabolism. Wiley Interdisciplinary Reviews RNA 1(3): 415-431. doi: 10.1002/wrna.39

Herrera, J.E., Savkur, R., and Olson, M.O. 1995. The ribonuclease activity of nucleolar protein B23. Nucleic Acids Research 23(19): 3974-3979. doi:

Hingorani, K., Szebeni, A., and Olson, M.O.J. 2000. Mapping the functional domains of nucleolar protein B23. Journal of Biological Chemistry 275(32): 24451-24457. doi: 10.1074/jbc.M003278200.

Hinz, J.M., and Czaja, W. 2015. Facilitation of base excision repair by chromatin remodeling. DNA Repair (Amst) 36: 91-97. doi: 10.1016/j.dnarep.2015.09.011.

Hovanessian, A.G., Soundaramourty, C., Khoury, D.E., Nondier, I., Svab, J. and Krust, B. 2010. Surface expressed nucleolin is constantly induced in tumor cells to mediate calcium-dependent ligand internalization. PLoS One 5(12): e15787. doi: 10.1371/journal.pone.0015787.

Hsu, T. I.; Lin, S. C.; Lu, P. S.; Chang, W. C.; Hung, C. Y.; Yeh, Y. M.; Su, W. C.; Liao, P. C. and Hung, J. J. 2015. MMP7-mediated cleavage of nucleolin at Asp255 induces MMP9 expression to promote tumor malignancy. Oncogene 34(7): 826-837. doi: 10.1038/onc.2014.22.

Huang, N., Negi, S., Szebeni, A., and Olson, M.O.J. 2005. Protein NPM3 interacts with the multifunctional nucleolar protein B23/nucleophosmin and inhibits ribosome biogenesis. J Biol Chem 280(7): 5496-5502. doi: $10.1074 /$ jbc.M407856200. 
Ikura, T.; Tashiro, S.; Kakino, A.; Shima, H.; Jacob, N.; Amunugama, R.; Yoder, K.; Izumi, S.; Kuraoka, I.; Tanaka, K.; Kimura, H.; Ikura, M.; Nishikubo, S.; Ito, T.; Muto, A.; Miyagawa, K.; Takeda, S.; Fishel, R.; Igarashi, K. and Kamiya, K. 2007. DNA damage-dependent acetylation and ubiquitination of H2AX enhances chromatin dynamics. Molecular and Cellular Biology 27(20): 7028-7040. doi: 10.1128/mcb.00579-07. Indig, F.E., Rybanska, I., Karmakar, P., Devulapalli, C., Fu, H., Carrier, F., and Bohr, V.A. 2012. Nucleolin inhibits G4 oligonucleotide unwinding by Werner helicase. PLoS One 7(6): e35229. doi: 10.1371/journal.pone.0035229. Ishikawa, F., Matunis, M.J., Dreyfuss, G., and Cech, T.R. 1993. Nuclear proteins that bind the pre-mRNA 3' splice site sequence $r(U U A G / G)$ and the human telomeric DNA sequence $d(T T A G G G) n$. Molecular and Cellular Biology 13(7): 4301-4310. doi: 10.1128/MCB.13.7.4301.

James, A., Wang, Y., Raje, H., Rosby, R., and DiMario, P. 2014. Nucleolar stress with and without p53. Nucleus 5(5): 402-426. doi: 10.4161/nucl.32235.

Jeggo, P.A., and Downs, J.A. 2014. Roles of chromatin remodellers in DNA double strand break repair. Experimental Cell Research 329(1): 69-77. doi: 10.1016/j.yexcr.2014.09.023.

Jobert, L., Skjeldam, Hanne K., Dalhus, B., Galashevskaya, A., Vågbø, Cathrine B., Bjørås, M., and Nilsen, H. 2013. The human base excision repair enzyme SMUG1 directly interacts with DKC1 and contributes to RNA quality control. Molecular Cell 49(2): 339-345. doi: 10.1016/j.molcel.2012.11.010.

Kakarougkas, A., and Jeggo, P.A. 2014. DNA DSB repair pathway choice: an orchestrated handover mechanism. British Journal of Radiology 87(1035): 20130685. doi: 10.1259/bjr.20130685.

Kim, J.-A., and Haber, J.E. 2009. Chromatin assembly factors Asf1 and CAF-1 have overlapping roles in deactivating the DNA damage checkpoint when DNA repair is complete. Proceedings of the National Academy of Sciences of the United States of America 106(4): 1151-1156. doi:

10.1073/pnas.0812578106. 
Kim, K., Dimitrova, D.D., Carta, K.M., Saxena, A., Daras, M., and Borowiec, J.A. 2005. Novel checkpoint response to genotoxic stress mediated by nucleolin-replication protein A complex formation. Molecular and Cellular Biology 25(6): 2463-2474. doi: 10.1128/MCB.25.6.2463-2474.2005.

Kim, Y., Kim, H.D., and Kim, J. 2013. Cytoplasmic ribosomal protein S3 (rpS3) plays a pivotal role in mitochondrial DNA damage surveillance. Biochimica et Biophysica Acta 1833(12): 2943-2952. doi: 10.1016/j.bbamcr.2013.07.015.

Kobayashi, J., Fujimoto, H., Sato, J., Hayashi, I., Burma, S., Matsuura, S., Chen, D.J., and Komatsu, K. 2012. Nucleolin participates in DNA double-strand break-induced damage response through MDC1dependent pathway. PLoS One 7(11): e49245.

Koike, A., Nishikawa, H., Wu, W., Okada, Y., Venkitaraman, A.R., and Ohta, T. 2010. Recruitment of phosphorylated NPM1 to sites of DNA damage through RNF8-dependent ubiquitin conjugates. Cancer Research 70(17): 6746-6756. 10.1158/0008-5472.CAN-10-0382.

Kowalczykowski, S.C. 2015. An overview of the molecular mechanisms of recombinational DNA repair. Cold Spring Harbor Perspectives in Biology 7(11). doi: 10.1101/cshperspect.a016410.

Kurki, S., Peltonen, K., Latonen, L., Kiviharju, T.M., Ojala, P.M., Meek, D., and Laiho, M. 2004. Nucleolar protein NPM interacts with HDM2 and protects tumor suppressor protein p53 from HDM2-mediated degradation. Cancer Cell 5(5): 465-475. doi: 10.1016/S1535-6108(04)00110-2.

Lamaye, F., Galliot, S., Alibardi, L., Lafontaine, D.L.J., and Thiry, M. 2011. Nucleolar structure across evolution: the transition between bi- and tricompartmentalized nucleoli lies within the class Reptilia. Journal of Structural Biology 174(2): 352-359. doi: 10.1016/j.jsb.2011.02.003.

Lan, L., Ui, A., Nakajima, S., Hatakeyama, K., Hoshi, M., Watanabe, R., Janicki, S.M., Ogiwara, H., Kohno, T., Kanno, S., and Yasui, A. 2010. The ACF1 complex is required for DNA double-strand break repair in human cells. Molecular Cell 40(6): 976-987. doi: 10.1016/j.molcel.2010.12.003. 
Lee, H.H., Kim, H.S., Kang, J.Y., Lee, B.I., Ha, J.Y., Yoon, H.J., Lim, S.O., Jung, G., and Suh, S.W. 2007. Crystal structure of human nucleophosmin-core reveals plasticity of the pentamer-pentamer interface. Proteins 69(3): 672-678. doi: 10.1002/prot.21504.

Lee, S.Y., Park, J.H., Kim, S., Park, E.J., Yun, Y., and Kwon, J. 2005. A proteomics approach for the identification of nucleophosmin and heterogeneous nuclear ribonucleoprotein $\mathrm{C} 1 / \mathrm{C} 2$ as chromatinbinding proteins in response to DNA double-strand breaks. Biochemistry Journal 388(1): 7-15. doi: 10.1042/BJ20042033.

Leung, A.K., Trinkle-Mulcahy, L., Lam, Y.W., Andersen, J.S., Mann, M., and Lamond, A.I. 2006. NOPdb: nucleolar proteome database. Nucleic Acids Research 34(Database issue): D218-220. doi: 10.1093/nar/gkj004.

Li, G.M. 2014. New insights and challenges in mismatch repair: getting over the chromatin hurdle. DNA Repair (Amst) 19: 48-54. doi: 10.1016/j.dnarep.2014.03.027.

Li, Y.P., Busch, R.K., Valdez, B.C., and Busch, H. 1996. C23 interacts with B23, a putative nucleolarlocalization-signal-binding protein. European Journal of Biochemistry 237(1): 153-158. doi: 10.1111/j.1432-1033.1996.0153n.

Lim, K.H., Park, J.J., Gu, B.H., Kim, J.O., Park, S.G., and Baek, K.H. 2015. HAUSP-nucleolin interaction is regulated by p53-Mdm2 complex in response to DNA damage response. Science Reports 5: 12793. doi: 10.1038/srep12793.

Lin, C.Y., Tan, B.C.-M., Liu, H., Shih, C.-J., Chien, K.-Y., Lin, C.-L., and Yung, B.Y.-M. 2010. Dephosphorylation of nucleophosmin by PP1 $\beta$ facilitates pRB binding and consequent E2F1-dependent DNA repair. Molecular Biology of the Cell 21(24): 4409-4417. doi: 10.1091/mbc.E10-03-0239. Lin, T., Ibrahim, W., Peng, C.Y., Finegold, M.J., and Tsai, R.Y. 2013. A novel role of nucleostemin in maintaining the genome integrity of dividing hepatocytes during mouse liver development and regeneration. Hepatology 58(6): 2176-2187. doi: 10.1002/hep.26600. 
Lindahl, T. 1993. Instability and decay of the primary structure of DNA. Nature 362(6422): 709-715. doi: 10.1038/362709a0.

Lirussi, L., Antoniali, G., Vascotto, C., D'Ambrosio, C., Poletto, M., Romanello, M., Marasco, D., Leone, M., Quadrifoglio, F., Bhakat, K.K., Scaloni, A., and Tell, G. 2012. Nucleolar accumulation of APE1 depends on charged lysine residues that undergo acetylation upon genotoxic stress and modulate its BER activity in cells. Molecular Biology of the Cell 23(20): 4079-4096. doi: 10.1091/mbc.E12-04-0299.

Liu, H., Deng, X., Shyu, Y.J., Li, J.J., Taparowsky, E.J., and Hu, C.D. 2006. Mutual regulation of c-Jun and ATF2 by transcriptional activation and subcellular localization. EMBO Journal 25(5): 1058-1069. doi: 10.1038/sj.emboj.7601020.

Liu, X., Liu, Z., Jang, S.-W., Ma, Z., Shinmura, K., Kang, S., Dong, S., Chen, J., Fukasawa, K., and Ye, K. 2007. Sumoylation of nucleophosmin/B23 regulates its subcellular localization, mediating cell proliferation and survival. Proceedings of the National Academy of Sciences of the United States of America 104(23): 9679-9684. doi: 10.1073/pnas.0701806104.

Lu, L.-Y.; Wu, J.; Ye, L.; Gavrilina, G. B.; Saunders, T. L. and Yu, X. 2010. RNF8-dependent histone modifications regulate nucleosome removal during spermatogenesis. Developmental Cell 18(3): 371384. doi: 10.1016/j.devcel.2010.01.010.

Maggi, L.B., Kuchenruether, M., Dadey, D.Y.A., Schwope, R.M., Grisendi, S., Townsend, R.R., Pandolfi, P.P., and Weber, J.D. 2008. Nucleophosmin serves as a rate-limiting nuclear export chaperone for the mammalian ribosome. Molecular and Cellular Biology 28(23): 7050-7065. doi: 10.1128/mcb.01548-07. Marteijn, J.A., Lans, H., Vermeulen, W., and Hoeijmakers, J.H.J. 2014. Understanding nucleotide excision repair and its roles in cancer and ageing. Nature Reviews Molecular Cell Biology 15(7): 465-481. doi: 10.1038/nrm3822.

Meng, L., Lin, T., Peng, G., Hsu, J.K., Lee, S., Lin, S.-Y., and Tsai, R.Y.L. 2013. Nucleostemin deletion reveals an essential mechanism that maintains the genomic stability of stem and progenitor cells. 
Proceedings of the National Academy of Sciences of the United States of America 110(28): 11415-11420. doi: 10.1073/pnas.1301672110.

Mitrea, D.M., Grace, C.R., Buljan, M., Yun, M.-K., Pytel, N.J., Satumba, J., Nourse, A., Park, C.-G., Madan Babu, M., White, S.W., and Kriwacki, R.W. 2014. Structural polymorphism in the N-terminal oligomerization domain of NPM1. Proceedings of the National Academy of Sciences of the United States of America 111(12): 4466-4471. doi: 10.1073/pnas.1321007111.

Mitrea, D.M., Cika, J.A., Guy, C.S., Ban, D., Banerjee, P.R., Stanley, C.B., Nourse, A., Deniz, A.A., and Kriwacki, R.W. 2016. Nucleophosmin integrates within the nucleolus via multi-modal interactions with proteins displaying R-rich linear motifs and rRNA. eLife 5. doi: 10.7554/eLife.13571.

Moore, H.M., Bai, B., Boisvert, F.-M., Latonen, L., Rantanen, V., Simpson, J.C., Pepperkok, R., Lamond, A.I., and Laiho, M. 2011. Quantitative proteomics and dynamic imaging of the nucleolus reveal distinct responses to UV and ionizing radiation. Molecular and Cellular Proteomics 10(10): M111.009241. doi: 10.1074/mcp.M111.009241.

Murano, K., Okuwaki, M., Hisaoka, M., and Nagata, K. 2008. Transcription regulation of the rRNA gene by a multifunctional nucleolar protein, B23/nucleophosmin, through its histone chaperone activity. Molecular and Cellular Biology 28(10): 3114-3126. doi: 10.1128/MCB.02078-07. Namboodiri, V.M.H., Dutta, S., Akey, I.V., Head, J.F., and Akey, C.W. 2003. The crystal structure of Drosophila NLP-core provides insight into pentamer formation and histone binding. Structure 11(2): 175186. doi: 10.1016/S0969-2126(03)00007-8.

Namboodiri, V.M., Akey, I.V., Schmidt-Zachmann, M.S., Head, J.F., and Akey, C.W. 2004. The structure and function of Xenopus NO38-core, a histone chaperone in the nucleolus. Structure 12(12): 2149-2160. doi: 10.1016/j.str.2004.09.017.

Nisole, S., Said, E.A., Mische, C., Prevost, M.-C., Krust, B., Bouvet, P., Bianco, A., Briand, J.-P., Hovanessian, A.G. 2002. The anti-HIV pentameric pseudopeptide HB-19 binds the C-terminal end of 
nucleolin and prevents anchorage of virus particles in the plasma membrane of target cells. Journal of Biological Chemistry 277(23): 20877-20886. doi: 10.1074/jbc.M110024200.

Ochi, T., Wu, Q., and Blundell, T.L. 2014. The spatial organization of non-homologous end joining: From bridging to end joining. DNA Repair (Amst) 17: 98-109. doi: 10.1016/j.dnarep.2014.02.010.

Okuda, M., Horn, H.F., Tarapore, P., Tokuyama, Y., Smulian, A.G., Chan, P.K., Knudsen, E.S., Hofmann, I.A., Snyder, J.D., Bove, K.E., and Fukasawa, K. 2000. Nucleophosmin/B23 is a target of CDK2/cyclin E in centrosome duplication. Cell 103(1): 127-140. doi: 10.1016/S0092-8674(00)00093-3.

Okuwaki, M., Matsumoto, K., Tsujimoto, M., and Nagata, K. 2001. Function of nucleophosmin/B23, a nucleolar acidic protein, as a histone chaperone. FEBS Letters 506(3): 272-276. doi: 10.1016/S00145793(01)02939-8.

Onikubo, T., Nicklay, J.J., Xing, L., Warren, C., Anson, B., Wang, W.-L., Burgos, E.S., Ruff, S.E., Shabanowitz, J., Cheng, R.H., Hunt, D.F., Shechter, D. 2015. Developmentally regulated posttranslational modification of nucleoplasmin controls histone sequestration and deposition. Cell Reports 10(10): 1735-1748. doi: 10.1016/j.celrep.2015.02.038.

Orrick, L.R., Olson, M.O.J., and Busch, H. 1973. Comparison of nucleolar proteins of normal rat liver and Novikoff hepatoma ascites cells by two-dimensional polyacrylamide gel electrophoresis. Proceedings of the National Academy of Sciences of the United States of America 70(5): 1316-1320. doi:

10.1073/pnas.70.5.1316.

Palaniswamy, V., Moraes, K.C.M., Wilusz, C.J., and Wilusz, J. 2006. Nucleophosmin is selectively deposited on mRNA during polyadenylation. Nature Structural and Molecular Biology 13(5): 429-435. doi: $10.1038 / \mathrm{nsmb} 1080$.

Pasternack, M. S.; Bleier, K. J. and Mclnerney, T. N. 1991. Granzyme A binding to target cell proteins: Granzyme A binds to and cleaves nucleolin in vitro. Journal of Biological Chemistry 266(22): 1470314708. 
Pederson, T. 2011. The nucleolus. Cold Spring Harbor Perspectives in Biology 3(3). doi:

10.1101/cshperspect.a000638.

Platonova, O., Akey, I.V., Head, J.F., and Akey, C.W. 2011. Crystal structure and function of human nucleoplasmin (NPM2): a histone chaperone in oocytes and embryos. Biochemistry 50(37): 8078-8089. doi: $10.1021 /$ bi2006652.

Poletto, M., Lirussi, L., Wilson, D.M., 3rd, and Tell, G. 2014. Nucleophosmin modulates stability, activity, and nucleolar accumulation of base excision repair proteins. Molecular Biology of the Cell 25(10): 16411652. doi: 10.1091/mbc.E13-12-0717.

Poletto, M., Malfatti, E., Dorjsuren, D., Scognamiglio, P.L., Marasco, D., Vascotto, C., Jadhav, A., Maloney, D.J., Wilson, D.M., III, Simeonov, A., and Tell, G. 2015. Inhibitors of the apurinic/apyrimidinic endonuclease 1 (APE1)/nucleophosmin (NPM1) interaction that display anti-tumor properties. Molecular Carcinogenesis (in press). doi: 10.1002/mc.22313.

Prakash, A., and Borgstahl, G.E. 2012. The structure and function of replication protein A in DNA replication. Sub-Cellular Biochemistry 62: 171-196. doi: 10.1007/978-94-007-4572-8_10.

Rathke, C., Baarends, W.M., Awe, S., and Renkawitz-Pohl, R. 2014. Chromatin dynamics during spermiogenesis. Biochimica et Biophysica Acta (BBA) - Gene Regulatory Mechanisms 1839(3): 155-168. doi: 10.1016/j.bbagrm.2013.08.004.

Rickards, B., Flint, S.J., Cole, M.D., and LeRoy, G. 2007. Nucleolin is required for RNA polymerase I transcription in vivo. Molecular and Cellular Biology 27(3): 937-948. doi: 10.1128/mcb.01584-06. Sato, K., Hayami, R., Wu, W., Nishikawa, T., Nishikawa, H., Okuda, Y., Ogata, H., Fukuda, M., and Ohta, T. 2004. Nucleophosmin/B23 Is a candidate substrate for the BRCA1-BARD1 ubiquitin ligase. Journal of Biological Chemistry 279(30): 30919-30922. doi: 10.1074/jbc.C400169200.

Savkur, R.S., and Olson, M.O. 1998. Preferential cleavage in pre-ribosomal RNA byprotein B23 endoribonuclease. Nucleic Acids Research 26(19): 4508-4515. doi: 10.1093/nar/26.19.4508. 
Saxena, A., Rorie, C.J., Dimitrova, D., Daniely, Y., and Borowiec, J.A. 2006. Nucleolin inhibits Hdm2 by multiple pathways leading to p53 stabilization. Oncogene 25(55): 7274-7288. doi: 10.1038/sj.onc.1209714.

Schmidt-Zachmann, M.S., and Nigg, E.A. 1993. Protein localization to the nucleolus: a search for targeting domains in nucleolin. Journal of Cell Science 105 (3): 799-806. doi:

10.1016/j.biocel.2010.01.011.

Scott, D.D., Trahan, C., Zindy, P.J., Aguilar, C.L., Delubac, M.Y., Wei, K.E., and Oeffinger, M.H. 2016. Nol12 is a multifunctional endonuclease at the nexus of RNA and DNA metabolism. bioRxiv doi: http://dx.doi.org/10.1101/043935; NAR In Revision.

Seinsoth, S., UhImann-Schiffler, H., and Stahl, H. 2003. Bidirectional DNA unwinding by a ternary complex of T antigen, nucleolin and topoisomerase I. EMBO Reports 4(3): 263-268. doi:

10.1038/sj.embor.embor770.

Sekhar, K.R., Reddy, Y.T., Reddy, P.N., Crooks, P.A., Venkateswaran, A., McDonald, W.H., Geng, L., Sasi, S., Van Der Waal, R.P., Roti, J.L., Salleng, K.J., Rachakonda, G., and Freeman, M.L. 2011. The novel chemical entity YTR107 inhibits recruitment of nucleophosmin to sites of DNA damage, suppressing repair of DNA double-strand breaks and enhancing radiosensitization. Clinical Cancer Research 17(20): 6490-6499. doi: 10.1158/1078-0432.CCR-11-1054.

Suzuki, A., Kogo, R., Kawahara, K., Sasaki, M., Nishio, M., Maehama, T., Sasaki, T., Mimori, K., and Mori, M. 2012. A new PICTure of nucleolar stress. Cancer Science 103(4): 632-637. doi: 10.1111/j.13497006.2012.02219.x.

Swaminathan, V., Kishore, A.H., Febitha, K.K., and Kundu, T.K. 2005. Human histone chaperone nucleophosmin enhances acetylation-dependent chromatin transcription. Molecular and Cellular Biology 25(17): 7534-7545. doi: 10.1128/MCB.25.17.7534-7545.2005. 
Szebeni, A., and Olson, M.O.J. 1999. Nucleolar protein B23 has molecular chaperone activities. Protein Science 8(4): 905-912. doi: 10.1110/ps.8.4.905.

Tajrishi, M.M., Tuteja, R., and Tuteja, N. 2011. Nucleolin: the most abundant multifunctional phosphoprotein of nucleolus. Communicative and Integrative Biology 4(3): 267-275. doi: 10.4161/cib.4.3.14884.

Takagi, M., Absalon, M.J., McLure, K.G., and Kastan, M.B. 2005. Regulation of p53 translation and induction after DNA damage by ribosomal protein L26 and nucleolin. Cell 123(1): 49-63. doi: 10.1016/j.cell.2005.07.034.

Taneva, S.G., Bañuelos, S., Falces, J., Arregi, I., Muga, A., Konarev, P.V., Svergun, D.I., Velázquez-Campoy, A., and Urbaneja, M.A. 2009. A mechanism for histone chaperoning activity of nucleoplasmin: thermodynamic and structural models. J Mol Biol 393(2): 448-463. doi: 10.1016/j.jmb.2009.08.005. Tarapore, P., Okuda, M., and Fukasawa, K. 2002. A mammalian in vitro centriole duplication system: evidence for involvement of CDK2/cyclin E and nucleophosmin/B23 in centrosome duplication. Cell Cycle 1(1): 75-81. doi: 10.4161/cc.1.1.103.

Tarapore, P., Shinmura, K., Suzuki, H., Tokuyama, Y., Kim, S.-H., Mayeda, A., and Fukasawa, K. 2006. Thr199 phosphorylation targets nucleophosmin to nuclear speckles and represses pre-mRNA processing. FEBS Letters 580(2): 399-409. doi: 10.1016/j.febslet.2005.12.022.

Tham, K.C., Kanaar, R., and Lebbink, J.H. 2016. Mismatch repair and homeologous recombination. DNA Repair (Amst) 38: 75-83. doi: 10.1016/j.dnarep.2015.11.010.

Thiry, M., and Lafontaine, D.L.J. 2005. Birth of a nucleolus: the evolution of nucleolar compartments. Trends in Cell Biology 15(4): 194-199. doi: 10.1016/j.tcb.2005.02.007.

Thyagarajan, B., Lundberg, R., Rafferty, M., and Campbell, C. 1998. Nucleolin promotes homologous DNA pairing in vitro. Somatic Cell and Molecular Genetics 24(5): 263-272. doi:

10.1023/B:SCAM.0000007129.98789.1f. 
Tokuyama, Y., Horn, H.F., Kawamura, K., Tarapore, P., and Fukasawa, K. 2001. Specific phosphorylation of nucleophosmin on Thr(199) by cyclin-dependent kinase 2-cyclin E and its role in centrosome duplication. Journal of Biological Chemistry 276(24): 21529-21537. doi: 10.1074/jbc.M100014200.

Tsai, R.Y., and Pederson, T. 2014. Connecting the nucleolus to the cell cycle and human disease. FASEB Journal 28(8): 3290-3296. doi: 10.1096/fj.14-254680.

Turner, A.J., Knox, A.A., Prieto, J.L., McStay, B., and Watkins, N.J. 2009. A novel small-subunit processome assembly intermediate that contains the U3 snoRNP, nucleolin, RRP5, and DBP4. Molecular and Cellular Biology 29(11): 3007-3017. doi: 10.1128/mcb.00029-09.

Tuteja, N., Huang, N.W., Skopac, D., Tuteja, R., Hrvatic, S., Zhang, J., Pongor, S., Joseph, G., Faucher, C., Amalric, F. and Falaschi, A. 1995. Human DNA helicase IV is nucleolin, an RNA helicase modulated by phosphorylation. Gene 160(2): 143-148. doi: 10.1016/0378-1119(95)00207-M.

Vanderwaal, R.P., Maggi, L.B., Weber, J.D., Hunt, C.R., and Roti Roti, J.L. 2009. Nucleophosmin redistribution following heat shock: a role in heat-induced radiosensitization. Cancer Research 69(16): 6454-6462. doi: 10.1158/0008-5472.CAN-08-4896.

Vanderwaal, R.P., and Roti Roti, J.L. 2004. Heat induced 'masking' of redox sensitive component(s) of the DNA-nuclear matrix anchoring complex. International Journal of Hyperthermia 20(2): 234-239. doi: $10.1080 / 02656730310001627704$.

Vascotto, C., Fantini, D., Romanello, M., Cesaratto, L., Deganuto, M., Leonardi, A., Radicella, J.P., Kelley, M.R., D'Ambrosio, C., Scaloni, A., Quadrifoglio, F., and Tell, G. 2009. APE1/Ref-1 interacts with NPM1 within nucleoli and plays a role in the rRNA quality control process. Molecular and Cellular Biology 29(7): 1834-1854. doi: 10.1128/MCB.01337-08.

Vascotto, C., Lirussi, L., Poletto, M., Tiribelli, M., Damiani, D., Fabbro, D., Damante, G., Demple, B., Colombo, E., and Tell, G. 2014. Functional regulation of the apurinic/apyrimidinic endonuclease 1 by nucleophosmin: impact on tumor biology. Oncogene 33(22): 2876-2887. doi: 10.1038/onc.2013.251. 
Vassin, V.M., Wold, M.S., and Borowiec, J.A. 2004. Replication protein A (RPA) phosphorylation prevents RPA association with replication centers. Molecular and Cellular Biology 24(5): 1930-1943. doi: 10.1128/MCB.24.5.1930-1943.2004.

Venema, J. and Tollervey, D. 1999. Ribosome synthesis in Saccharomyces cerevisiae. Annual Review of Genetics 33: 261-311. doi: 10.1146/annurev.genet.33.1.261.

Wade, M., Li, Y.-C., and Wahl, G.M. 2013. MDM2, MDMX and p53 in oncogenesis and cancer therapy. Nature Reviews Cancer 13(2): 83-96. doi: 10.1038/nrc3430.

Wang, H.-F., Takenaka, K., Nakanishi, A., and Miki, Y. 2011. BRCA2 and nucleophosmin coregulate centrosome amplification and form a complex with the Rho effector kinase ROCK2. Cancer Research 71(1): 68-77. doi: 10.1158/0008-5472.can-10-0030.

Wang, W., Budhu, A., Forgues, M., and Wang, X.W. 2005. Temporal and spatial control of nucleophosmin by the Ran-Crm1 complex in centrosome duplication. Nature Cell Biology 7(8): 823-830. doi: $10.1038 /$ ncb1282.

Wang, Y., Guan, J., Wang, H., Wang, Y., Leeper, D., and Iliakis, G. 2001. Regulation of DNA replication after heat shock by replication protein A-nucleolin interactions. Journal of Biological Chemistry 276(23): 20579-20588. doi: 10.1074/jbc.M100874200.

Warrener, P. and Petryshyn, R. 1991. Phosphorylation and proteolytic degradation of nucleolin from 3T3-F442A cells. Biochemical and Biophysical Research Communications 180(2): 716-723. doi: 10.1016/S0006-291X(05)81124-6.

Waters, L.S., Minesinger, B.K., Wiltrout, M.E., D'Souza, S., Woodruff, R.V., and Walker, G.C. 2009. Eukaryotic translesion polymerases and their roles and regulation in DNA damage tolerance. Microbiology and Molecular Biology Reviews 73(1): 134-154. doi: 10.1128/MMBR.00034-08. Woolford, J. L., Jr. and Baserga, S. J. 2013. Ribosome biogenesis in the yeast Saccharomyces cerevisiae. Genetics 195(3): 643-681. doi: 10.1534/genetics.113.153197. 
Wu, M.H., Chang, J.H., and Yung, B.Y. 2002a. Resistance to UV-induced cell-killing in nucleophosmin/B23 over-expressed NIH 3T3 fibroblasts: enhancement of DNA repair and up-regulation of PCNA in association with nucleophosmin/B23 over-expression. Carcinogenesis 23(1): 93-100. doi: 10.1093/carcin/23.1.93.

Wu, M.H., Chang, J.H., Chou, C.C., and Yung, B.Y. 2002b. Involvement of nucleophosmin/B23 in the response of HeLa cells to UV irradiation. International Journal of Cancer 97(3): 297-305. doi: 10.1002/ijc.1606.

Xia, X., Liu, S., Xiao, Z., Zhu, F., Song, N.Y., Zhou, M., Liu, B., Shen, J., Nagashima, K., Veenstra, T.D., Burkett, S., Datla, M., Willette-Brown, J., Shen, H., and Hu, Y. 2013. An IKKalpha-nucleophosmin axis utilizes inflammatory signaling to promote genome integrity. Cell Reports 5(5): 1243-1255. doi: 10.1016/j.celrep.2013.10.046.

Yamamori, T., DeRicco, J., Naqvi, A., Hoffman, T.A., Mattagajasingh, I., Kasuno, K., Jung, S.-B., Kim, C.-S., and Irani, K. 2010. SIRT1 deacetylates APE1 and regulates cellular base excision repair. Nucleic Acids Research 38(3): 832-845. doi: 10.1093/nar/gkp1039.

Yang, C., Kim, M.S., Chakravarty, D., Indig, F.E., and Carrier, F. 2009. Nucleolin binds to the proliferating cell nuclear antigen and inhibits nucleotide excision repair. Molecular and Cellular Pharmacology 1(3): 130-137. doi: 10.4255/mcpharmacol.09.17.

Yang, C., Maiguel, D.A., and Carrier, F. 2002. Identification of nucleolin and nucleophosmin as genotoxic stress-responsive RNA-binding proteins. Nucleic Acids Research 30(10): 2251-2260. doi: $10.1093 /$ nar/30.10.2251.

Yogev, O., Saadon, K., Anzi, S., Inoue, K., and Shaulian, E. 2008. DNA damage-dependent translocation of B23 and p19ARF is regulated by the Jun N-terminal kinase pathway. Cancer Research 68(5): 1398-1406. doi: 10.1158/0008-5472.can-07-2865. 
Yu, Y., Maggi, L.B., Brady, S.N., Apicelli, A.J., Dai, M.-S., Lu, H., and Weber, J.D. 2006. Nucleophosmin is essential for ribosomal protein L5 nuclear export. Molecular and Cellular Biology 26(10): 3798-3809. doi: 10.1128/MCB.26.10.3798-3809.2006.

Zhang, D., Liang, Y., Xie, Q., Gao, G., Wei, J., Huang, H., Li, J., Gao, J., and Huang, C. 2015. A novel posttranslational modification of nucleolin, SUMOylation at Lys-294, mediates arsenite-induced cell death by regulating gadd45 $\alpha$ mRNA stability. Journal of Biological Chemistry 290(8): 4784-4800. doi: 10.1074/jbc.M114.598219.

Ziv, O., Zeisel, A., Mirlas-Neisberg, N., Swain, U., Nevo, R., Ben-Chetrit, N., Martelli, M.P., Rossi, R., Schiesser, S., Canman, C.E., Carell, T., Geacintov, N.E., Falini, B., Domany, E., and Livneh, Z. 2014. Identification of novel DNA-damage tolerance genes reveals regulation of translesion DNA synthesis by nucleophosmin. Nature Communications 5: 5437. doi: 10.1038/ncomms6437. 


\section{Figure Legends}

Figure 1: Schematic of human NCL and NPM1 proteins indicating the relative positions of domains and post-translationally modified residues discussed in this review. Brackets beneath each protein indicate the reported minimal regions for interactions of each protein with its respective protein partners, where such domains have been reported; dotted brackets for NCL indicate that a region which is not absolutely required for the interaction but enhances the affinity of NCL for the target. Interaction surfaces were determined by truncation mutants and interaction screening in all cases as follows: NCL-NPM1 (Li et al. 1996); NCL-RAD50 (Goldstein et al. 2013); NCL-HDM2 and NCL-p53 (Bhatt et al. 2012); NCL-WRN (Indig et al. 2012); NCL-RPA (Kim et al. 2005); NCL-ssDNA (Hanakahi et al. 2000); NCL-G-quadruplex (González and Hurley 2010; Hanakahi et al. 1999); NPM1-p53 (Colombo et al. 2002); NPM1-H3/H4 (Okuwaki et al. 2001); NPM1-polyUb (Koike et al. 2010); and NPM1-APE1 (Vascotto et al. 2009).

Figure 2: Models for the coordination of DNA damage repair pathways in response to different damaging stimuli by NPM1 and NCL. The discussed regulatory activities of NPM1 (A) and NCL (B) are summarized with respect to their inducing DNA lesion. The upper grey box represents the nucleolus, while the lower, clear regions represent the nucleoplasm. Thick black arrows represent enhancement of translocation rate in the indicated direction, while dotted arrows indicate a proposed or disputed activity. Green arrows indicate a positive regulatory effect, while red, flat-faced arrows indicate an inhibitory action. Question marks indicate unknown activities or mechanisms. Small red circles indicate regulatory post-translational modifications (acetylation/phosphorylation). 


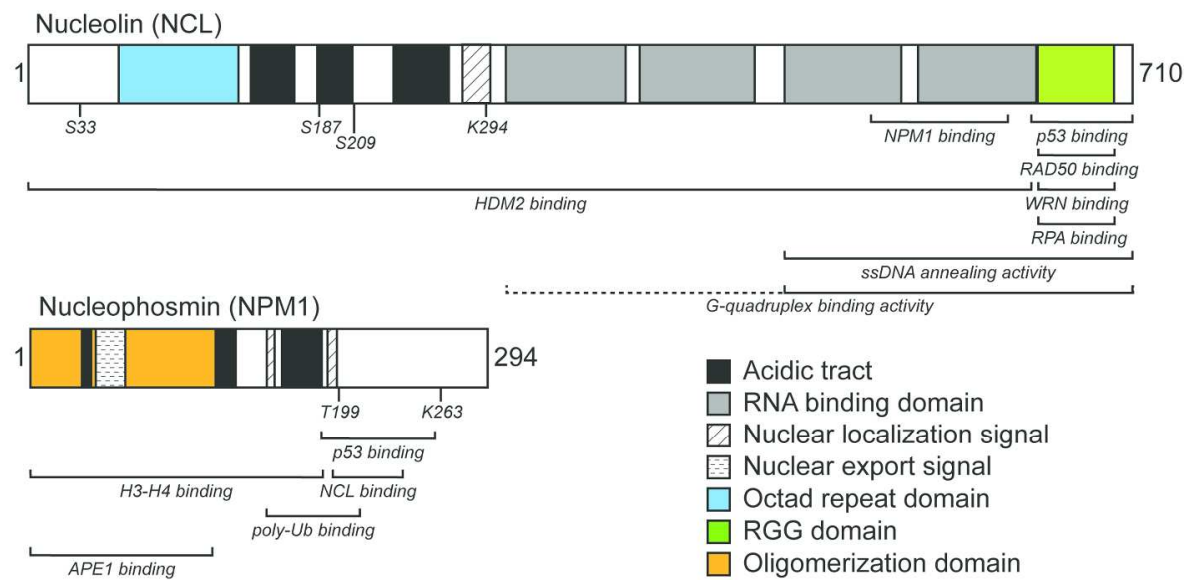

Scott and Oeffinger, Figure 1

Figure 1: Schematic of human NCL and NPM1 proteins indicating the relative positions of domains and posttranslationally modified residues discussed in this review. Brackets beneath each protein indicate the reported minimal regions for interactions of each protein with its respective protein partners, where such domains have been reported; dotted brackets for NCL indicate that a region which is not absolutely required for the interaction but enhances the affinity of NCL for the target. Interaction surfaces were determined by truncation mutants and interaction screening in all cases as follows: NCL-NPM1 (Li et al. 1996); NCL-RAD50 (Goldstein et al. 2013); NCL-HDM2 and NCL-p53 (Bhatt et al. 2012); NCL-WRN (Indig et al. 2012); NCL-RPA (Kim et al. 2005); NCL-ssDNA (Hanakahi et al. 2000); NCL-G-quadruplex (González and Hurley 2010; Hanakahi et al. 1999); NPM1-p53 (Colombo et al. 2002); NPM1-H3/H4 (Okuwaki et al. 2001); NPM1-polyUb (Koike et al. 2010); and NPM1-APE1 (Vascotto et al. 2009).

$194 \times 249 \mathrm{~mm}(300 \times 300 \mathrm{DPI})$ 
A
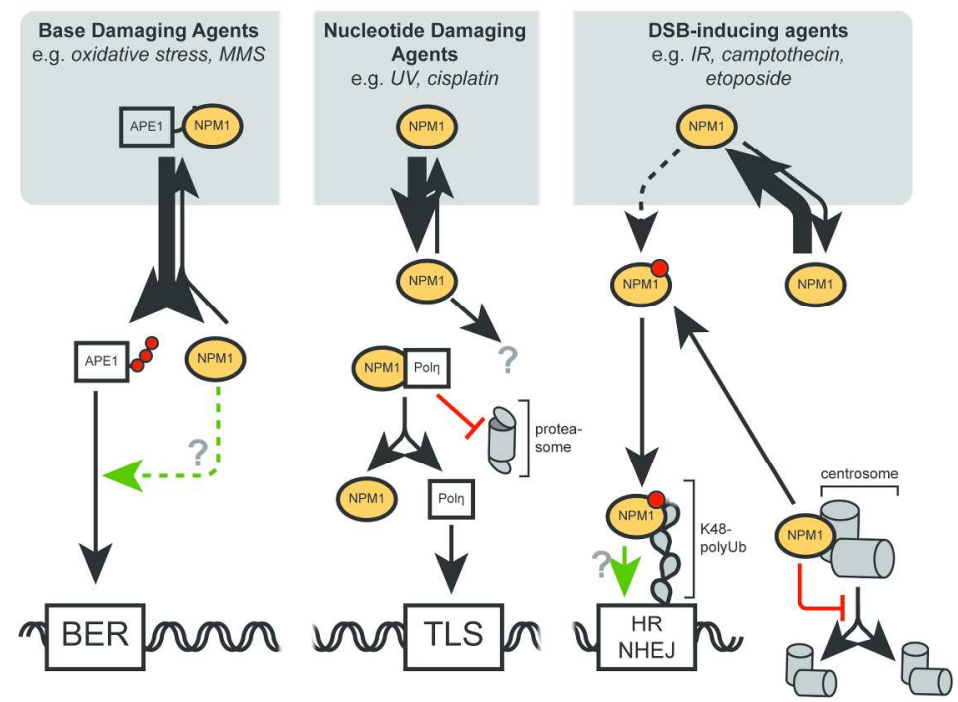

B
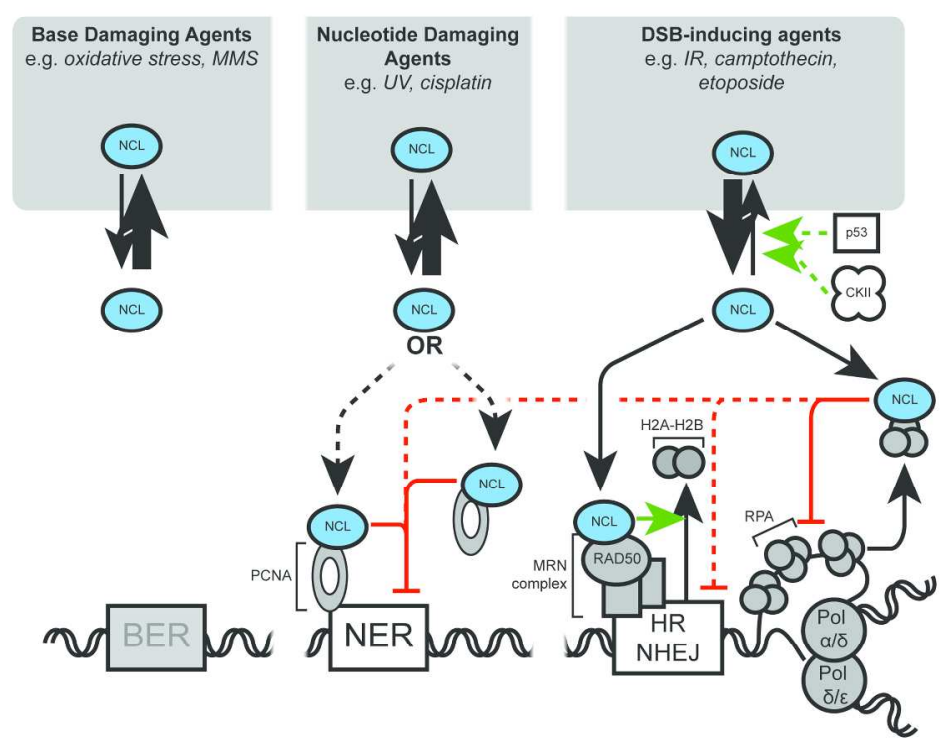

Scott and Oeffinger, Figure 2

Figure 2: Models for the coordination of DNA damage repair pathways in response to different damaging stimuli by NPM1 and NCL. The discussed regulatory activities of NPM1 (A) and NCL (B) are summarized with respect to their inducing DNA lesion. The upper grey box represents the nucleolus, while the lower, clear regions represent the nucleoplasm. Thick black arrows represent enhancement of translocation rate in the indicated direction, while dotted arrows indicate a proposed or disputed activity. Green arrows indicate a positive regulatory effect, while red, flat-faced arrows indicate an inhibitory action. Question marks indicate unknown activities or mechanisms. Small red circles indicate regulatory post-translational modifications

(acetylation/phosphorylation).

$182 \times 264 \mathrm{~mm}(300 \times 300 \mathrm{DPI})$ 